\title{
CALIBRACIÓN DE HIDRÓMETROS CON EL USO DE UN SISTEMA SEMI-AUTOMATIZADO DE AJUSTE Y DE ADQUISICIÓN DE DATOS
}

\author{
Olman Fernando Ramos Alfaro
}

\begin{abstract}
Resumen
Este artículo proporciona información sobre la implementación de mejoras realizadas al proceso de calibración de hidrómetros (densímetros de inmersión) en el Laboratorio de Densidad del LACOMET (Laboratorio Costarricense de Metrología).

La implementación de mejoras consistió en la definición e implementación de diferentes tareas tanto de investigación como de trabajo práctico entre las que se encuentran: definición del método de calibración (Método de Cuckow); definición de los métodos de ajuste del menisco formado entre el líquido de calibración y el hidrómetro; determinación de la necesidad de estabilizar el proceso de medición, definir el método de ajuste más consistente con la necesidad de estabilidad del sistema; determinación del uso de una varilla de vidrio para el ajuste; determinación del uso de un motor paso a paso para el proceso de ajuste; creación de un circuito de control manejable por medio del puerto paralelo de la PC; definición del acceso de equipos alternos por medio del puerto serial (RS232); desarrollo de un programa de control con LabVIEW® para el manejo de un motor paso a paso por medio del puerto paralelo de la PC y el uso del puerto serial para la captura de datos de los equipos de apoyo durante el proceso de calibración; creación de un panel frontal de control del proceso con capacidad de acceso a los diferentes equipos involucrados en la calibración; desarrollo de una tabla en el panel frontal para el control de los datos generados; planificación y presentación de la forma adecuada en la que se disponen los equipos involucrados en el proceso; por último, análisis de los resultados obtenidos por el proceso de calibración antes de implementar las mejoras y después de la implementación de ellas.

El uso de LabVIEW® como medio de control de todo el proceso de calibración de hidrómetros representa una mejora de importancia por la facilidad con la que la información es colectada y procesada, además la posibilidad de controlar el método de ajuste del menisco con el uso de una varilla de vidrio y un motor paso a paso ha mejorado el nivel de estabilidad del proceso y los tiempos de respuesta en el análisis de datos y en la emisión de los resultados finales.
\end{abstract}

Palabras clave: metrología, LACOMET, densidad de sólidos, hidrómetros, motores paso a paso, LabVIEW ${ }^{\circledR}$, modelo matemático, estimación de la incertidumbre.

\begin{abstract}
This article provides information about the implementation of improvements to the process of hydrometer calibration in the LACOMET Density Laboratory (Costa Rica Laboratory of Metrology).

The implementation of improvements was the definition and implementation of different tasks of both research and practice, these included: definition of the calibration method (Cuckow Method), defining the methods for adjustment of the meniscus formed between the liquid for calibration and the hydrometer, determination the need to stabilize the measurement process, determination of the method for adjustment of the meniscus consistent with need to stabilize the measurement process, determination of the use of a glass rod for adjustment, determine the use of a stepping motor for the adjustment process, create a manageable control circuit through the parallel port of the PC, define the access for other equipment through serial port (RS232), develop a program with LabVIEW ${ }^{\circledR}$ for control and monitoring of a stepping motor through the parallel port of the PC, and use the serial port to capture data support teams during the calibration process, create a front panel control of the process with access to the different teams involved in the calibration, develop a table on the front panel for the control of the generated data, plan and present the right way in which the equipments involved in the process will be located, finally, analyze the results of the calibration process before and after changes.

The use of LabVIEW ${ }^{\circledR}$ as a means of controlling the whole process of calibration of hydrometers represents a major improvement about the ease with which the information is collected and processed, furthermore, the possibility of controlling the method of adjustment of the meniscus with the use of a glass rod and a stepping motor has improved the level of stability of the process and response times in the data analysis and the issuance of the final results.
\end{abstract}

Keywords: metrology, LACOMET, density of solids, hydrometers, stepper motors, LabVIEW ${ }^{\circledR}$, mathematical model, estimation of uncertainty.

Recibido: 30 de marzo del 2009 • Aprobado: 6 de agosto del 2009. 


\section{INTRODUCCIÓN}

La Metrología, como la ciencia de las mediciones, utiliza métodos estandarizados de medición e instrumentos de medición conocidos y caracterizados. Distintas áreas de la ciencia y la técnica se han beneficiado con la mejora gradual en el campo de las mediciones al disponer de resultados más exactos y confiables. Aplicaciones simples y de bajo costo han logrado favorecer estas mejoras en los procesos de medición, mejoras que son cada vez más necesarias en un área donde hasta el mínimo aporte se torna importante.

Uno de los aspectos que más ha contribuido en la mejora de los procesos metrológicos ha sido la utilización de los medios de transmisión y adquisición de datos, estos, los más agilizados, sólidos, confiables e integrables que conjuntan diferentes equipos en una sola aplicación, son posibles al crear programas a la medida de las necesidades de cada laboratorio.

La integración de diferentes equipos para la adquisición de datos, junto con la posibilidad de manejar y controlar un proceso con el uso de una computadora se puede realizar con el uso del software LabVIEW ${ }^{\circledR}$, de National Instruments, el cual es una herramienta de programación de tipo gráfico de accesibilidad y manejo sencillo y efectivo.

Este artículo presenta las innovaciones y mejoras introducidas en el proceso de calibración de hidrómetros, con el uso del Método de Cuckow, al sustituir el método manual de ajuste del menisco a un método semiautomatizado en el Laboratorio de Densidad de LACOMET, el cual es el Laboratorio Costarricense de Metrología, ubicado en la Ciudad de la Investigación de la Universidad de Costa Rica. Además se presentan los resultados obtenidos al comparar calibraciones previas a la implementación de este nuevo sistema y posteriores a esta.

El objetivo principal de este artículo es proporcionar una herramienta de consulta con la cual otros laboratorios puedan mejorar sus métodos de calibración con el uso de materiales de bajo costo y de fácil acceso.

\section{METODOLOGÍA}

La calibración de un hidrómetro implica la preparación de un área adecuada en la cual puedan ser integrados varios equipos que tienen la finalidad de ser mecanismos de medición de las unidades que definen el modelo en estudio. Las metodologías de calibración tradicionales no han integrado medios modernos de adquisición de datos y de control de los equipos utilizados comúnmente en estos procesos, esto quiere decir que existen laboratorios de calibración que al realizar el proceso de calibración de hidrómetros registran datos a mano, siendo este su principal medio de registro y no utiliza otros medios de registro o control de los datos. Además realizan procesos de ajuste para la lectura de los equipos involucrados con herramientas manuales, esto representa un problema ya que algunas variables que son registradas no son consideradas en tiempo real, tal es el caso de la temperatura en el fluido de calibración la cual puede fluctuar muy rápidamente, por otro lado, existe la posibilidad de que el error invalide los datos registrados y los resultados obtenidos. Para mejorar el proceso de registro de los datos de calibración en tiempo real, junto con los medios de control de los equipos para la lectura de estos datos, se crearon e implementaron dos elementos que hasta este momento no habían sido utilizados en el proceso de calibración en estudio. El primer elemento fue la fabricación de un sistema electromecánico de ajuste para los niveles adecuados de lectura del menisco que se produce en el fluido de calibración por un hidrómetro, esto se explicará más adelante; el otro elemento fue el desarrollo de una aplicación en LabVIEW ${ }^{\circledR}$ que permitiera controlar tanto el sistema electromecánico fabricado, como todos los equipos necesarios para el desarrollo del proceso, esto con la finalidad primordial de registrar los datos obtenidos en tiempo real de todos estos equipos y almacenarlos como un reporte generado al final del proceso en una hoja de cálculo.

Con base en lo anterior se investigó el método usado para la calibración de hidrómetros y con esta base y las necesidades del mismo, se desarrollaron el sistema electromecánico y la aplicación para el control de dicho sistema. 


\subsection{Hidrómetros}

Un hidrómetro es un instrumento usado para la medición de la densidad de líquidos, construido por lo general en vidrio o en algún tipo de material incoloro. Se compone de un tubo delgado o cuello (vástago), con una escala graduada y un cuerpo en forma de bulbo alargado el cual contiene en el fondo un material de contrapeso, o lastre, de masa constante, por lo general plomo, permitiéndole flotar en posición vertical al ser sumergido en un líquido. Cuando el hidrómetro flota libremente dentro del líquido en estudio deja parte de su cuello, y por ende parte de la escala, expuesto al medio ambiente. La densidad será leída en la escala del cuello como una función de su altura de la parte sumergida y del empuje ejercido por el líquido en estudio sobre el hidrómetro (García, 2006).

Uno de los procesos más complejos en el área de densidad, por su proceso de ajuste del menisco, la variedad de equipos usados, los controles requeridos y la dificultad del método de lectura es la calibración de hidrómetros, también llamados densímetros de inmersión.

La comprensión clara del método con el cual se calibran los hidrómetros y las restricciones o parámetros de control que el mismo método establece, determina cuáles son los equipos necesarios y la forma en la cual puede ser controlado el proceso, es allí en donde pueden introducirse las mejoras al proceso.

\subsection{Calibración de hidrómetros}

La calibración de los hidrómetros se basa en el principio de Arquímedes donde el valor de masa del instrumento es evaluado tanto en el aire como en un líquido, preferiblemente de densidad conocida, con el cual sea posible verificar el comportamiento de la escala graduada del mismo al producirse un desplazamiento de este líquido, a este método se le conoce como el Método de Cuckow.

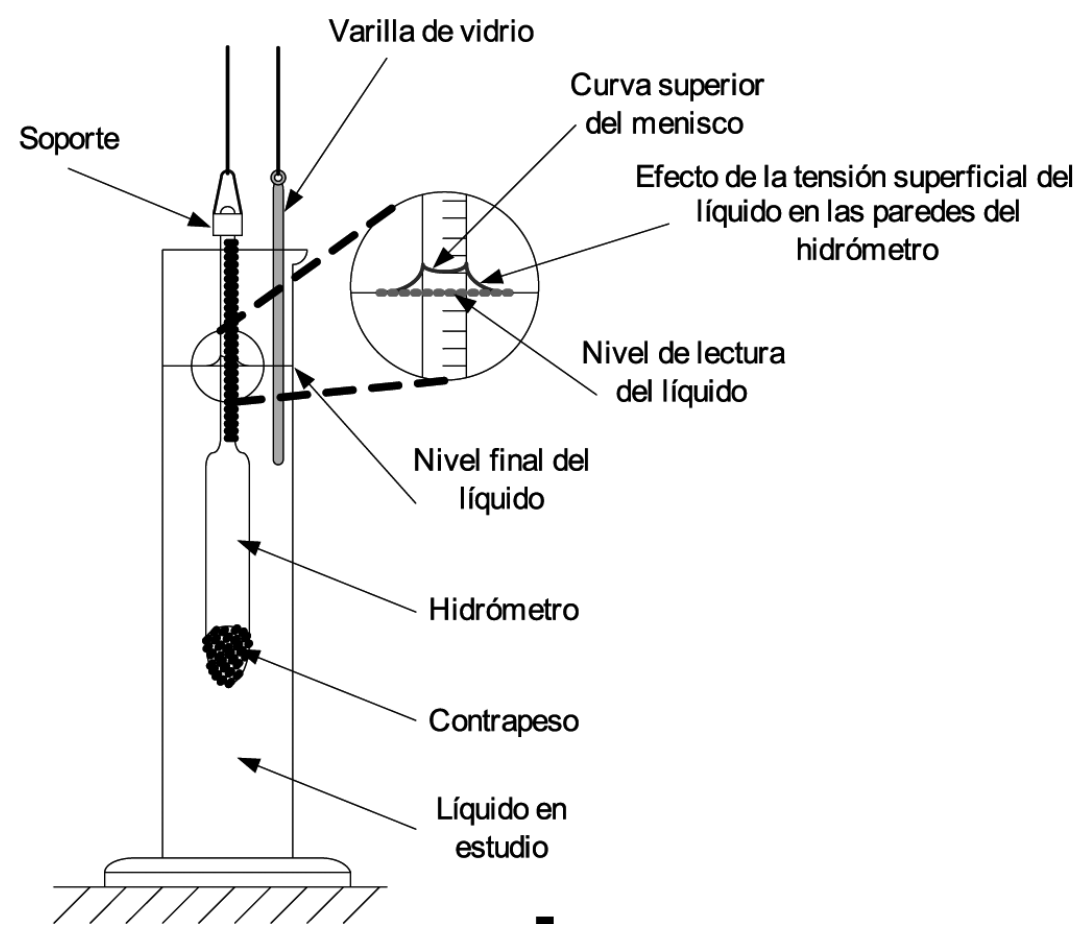

Figura 1. Hidrómetro sumergido. Fuente: (El autor). 


\subsubsection{Descripción del método}

El método de Cuckow consiste en colocar al hidrómetro un soporte en la parte superior del cuello de forma que este pueda suspenderse por debajo de una balanza analítica, esto define su masa al aire o masa seca. Posteriormente, se sumerge el hidrómetro dentro del líquido de densidad conocida en tres puntos diferentes de la escala a calibrar, preferiblemente $10 \%, 50 \%$, y 90 $\%$, y siempre colgando por debajo de la balanza para obtener su valor de masa sumergida o masa húmeda. La Figura 1 muestra la forma en la que el hidrómetro es sumergido en el líquido utilizado para la medición; del mismo modo, el recuadro de la Figura 1 muestra la forma como se comporta el líquido en presencia del material con el cual fue construido el hidrómetro, a esto se le llama menisco y es la curvatura producida por la tensión superficial del líquido ante el material del hidrómetro; el menisco produce un efecto de falsa lectura del valor de medición. Cuando se ajusta el líquido al nivel de medición adecuado se debe tener cuidado en obtener la lectura de forma correcta (Gupta, 2002; Santo \& Cáceres, 2006).

El procedimiento se desarrolla de la siguiente forma (Santo \& Cáceres, 2006; Becerra \& Sandoval, 2002):

\section{a. Masa del hidrómetro al aire}

1. Se mide el diámetro de cuello del hidrómetro tres veces en los puntos de la escala a calibrar $(10 \%, 50 \%$, y $90 \%)$, el promedio de las tres mediciones será el diámetro del punto a calibrar $(D)$.

2. Se coloca un soporte metálico al hidrómetro y se suspende colgado debajo de una balanza analítica que previamente ha sido tarada. Tomar al menos tres mediciones del valor de la masa, el promedio de estas mediciones será el valor de la masa del hidrómetro $\left(m_{0}\right)$.

3. Se seleccionan patrones de masa que en conjunto sumen un valor de masa cercano a la masa del hidrómetro $\left(m_{0}\right)$.

4. Se coloca el hidrómetro suspendido colgado por debajo de la balanza analítica y se anota el valor de la masa del hidrómetro, esta medición será el valor de la masa de referencia del hidrómetro al aire $\left(m_{l}\right)$.

5. Se retira el hidrómetro de la balanza y se colocan las pesas sobre ella. Se anota el valor de la masa de las pesas, esta medición será el valor de la masa de los patrones del hidrómetro al aire $\left(m_{\text {pesas }}^{\mathrm{c}}\right)$.

6. Se anota el valor de las condiciones ambientales en cada una de las mediciones, esto determinará el valor de la densidad del aire $\left(\rho_{\text {airel }}\right)$.

7. Se repiten los pasos 4,5 y 6 al menos cinco veces, el promedio de estas mediciones determinará el valor de la masa del hidrómetro al aire o masa seca $\left(m_{\text {aire }}\right)$ de la siguiente forma.

$m_{\text {aire }}=m_{\text {pesas } 1}^{c}\left(1-\frac{\rho_{\text {aire } 1}}{\rho_{\text {pesas }}}\right)+\Delta m_{1}$

En donde:

\begin{tabular}{|c|c|}
\hline$m_{\text {aire }}$ & $\begin{array}{l}\text { masa del hidrómetro debido a } \\
\text { la pesada en el aire (masa del } \\
\text { hidrómetro al aire) }\end{array}$ \\
\hline$m_{\text {pesas }}^{\mathrm{c}}$ & $\begin{array}{l}\text { masa convencional de los } \\
\text { patrones }\end{array}$ \\
\hline$\rho_{\text {airel }}$ & $\begin{array}{l}\text { densidad del aire medida en el } \\
\text { momento en el que se mide la } \\
\text { masa del hidrómetro en el aire }\end{array}$ \\
\hline$\rho_{\text {pesas }}$ & $\begin{array}{l}\text { densidad del material de las pesas } \\
\text { usadas como patrón }\end{array}$ \\
\hline$\Delta m_{1}$ & $\begin{array}{l}\text { diferencia en la lectura de la masa } \\
\left(m_{1}-m_{\text {pesasl }}^{\mathrm{c}}\right)\end{array}$ \\
\hline
\end{tabular}

b. Masa sumergida del hidrómetro

8. Se llena el recipiente que va a contener al hidrómetro con el líquido de medición hasta un volumen adecuado para que el hidrómetro flote libremente sin chocar con las paredes. Se colocan dentro del recipiente un instrumento para medir la temperatura del líquido y el hidrómetro y se dejan estabilizar al menos por ocho horas. 
9. Se suspende el hidrómetro sumergido por debajo de la balanza analítica, que previamente ha sido tarada, y se ajusta el menisco formado en el nivel superior horizontal del líquido de medición al punto de la escala que se va a medir, tal y como se muestra en la Figura 1. Se toman al menos tres mediciones del valor de la masa, el promedio de estas mediciones será el valor de la masa del hidrómetro $\left(m_{00}\right)$.

10. Se seleccionan patrones de masa que en conjunto sumen un valor de masa cercano a la masa del hidrómetro $\left(m_{00}\right)$.

11. Se coloca el hidrómetro sumergido suspendido por debajo de la balanza analítica y se anota el valor de la masa del hidrómetro, esta medición será el valor de la masa de referencia del hidrómetro sumergido $\left(m_{2}\right)$.

12. Se retira el hidrómetro de la balanza y se colocan las pesas sobre ella. Se anota el valor de la masa de las pesas, esta medición será el valor de la masa de los patrones del hidrómetro sumergido $\left(m_{\text {pesas } 2}\right)$.

13. Se anota el valor de las condiciones ambientales en cada una de las mediciones, esto determinará el valor de la densidad del aire $\left(\rho_{\text {aire }}\right)$.

14. Se repiten los pasos 11,12 y 13 al menos cinco veces, el promedio de estas mediciones determinará el valor de la masa sumergida del hidrómetro o masa húmeda $\left(\mathrm{m}_{L}\right)$ de la siguiente forma.

$m_{L}=m_{\text {pesas } 2}^{c}\left(1-\frac{\rho_{\text {aire } 2}}{\rho_{\text {pesas }}}\right)+\Delta m_{2}$

En donde:

$\mathrm{m}_{L} \quad$ masa del hidrómetro debida a la pesada en el líquido de medición (masa sumergida) $\mathrm{m}^{\mathrm{c}}$ pesas $2 \quad$ masa convencional de los patrones

$\rho_{\text {aire } 2}$ densidad del aire medida en el momento en el que se mide la masa sumergida del hidrómetro

$\rho_{\text {pesas }} \quad$ densidad del material de las pesas usadas como patrón

$\Delta m_{2} \quad$ diferencia en la lectura de la masa $\left(\mathrm{m}_{00}-\mathrm{m}_{\text {pesas }}\right)$

(La aplicación del método y el tema se puede ampliar en Gupta, 2002).

\subsubsection{Modelo matemático}

La forma de realizar este método con lleva la tarea de ajustar el menisco formado entre el material de cuello y el líquido de medición, de forma muy cuidadosa, al valor adecuado de la escala y esperar el momento adecuado en el cual la balanza logre condiciones de estabilidad; del mismo modo se debe tener un cuidadoso control sobre el líquido de medición de tal forma que no se produzcan cambios de temperatura que modifiquen el valor de la tensión superficial del líquido o que modifiquen el diámetro del cuello del hidrómetro, lo que produciría un cambio en la forma del menisco y por ende en la lectura. Sin embargo, ya que estos factores son difíciles de controlar es recomendable introducir correcciones que permitan cuantificar el valor final de la densidad en las condiciones en las cuales se realiza la medición.

El desarrollo de un modelo matemático del Método de Cuckow que incluya estas variaciones en las condiciones de calibración permite obtener resultados óptimos de calibración. El siguiente es el modelo matemático que describe el Método de Cuckow (ecuación 1, según Gupta, 2002; Santo \& Cáceres, 2006).

$$
\rho_{x}=\left(\frac{\left(\rho_{L}-\rho_{\text {aire }}\right)\left(m_{\text {aire }}+\frac{\pi D \gamma_{x}}{g}\right)}{\left(m_{\text {aire }}-m_{L}\right)\left(1-\frac{\left(\rho_{\text {airel }}-\rho_{\text {aire } 2}\right)}{\rho_{\text {pesas }}}\right)+\frac{\pi D \gamma_{L}}{g}}\right)\left[1+\beta\left(T_{L}-T_{0}\right)\right]+\rho_{\text {aire }}
$$


En donde:

$\rho_{x} \quad$ densidad del hidrómetro en el punto de lectura

$\rho_{L} \quad$ densidad del líquido de medición

$\rho_{\text {aire }} \quad$ densidad del aire

$\rho_{\text {airel densidad del aire medida en el }}$ momento en el que se mide la masa del hidrómetro en el aire

$\rho_{\text {aire } 2 \text { densidad del aire medida en el }}$ momento en el que se mide la masa del hidrómetro en el líquido

$\rho_{\text {pesas }}$ densidad del material de las pesas usadas como patrón

$m_{\text {aire }} \quad$ masa del hidrómetro debida a la pesada en el aire

$m_{L} \quad$ masa del hidrómetro debida a la pesada en el líquido de medición en el punto de lectura

$\pi \quad$ valor de pi

D diámetro del cuello del hidrómetro en el punto de lectura

$g \quad$ valor de aceleración de la gravedad

$\gamma_{x} \quad$ tensión superficial del líquido en el que se va a utilizar el hidrómetro

$\gamma_{L}$ tensión superficial del líquido de medición

$\beta \quad$ coeficiente volumétrico de expansión térmica del material con el que está hecho el hidrómetro

$T_{L} \quad$ temperatura del líquido de medición durante el proceso de calibración

$T_{0} \quad$ temperatura del líquido de medición durante el proceso de calibración

El objetivo del proceso de calibración de un hidrómetro es determinar la corrección de la escala graduada contra un valor dado de densidad del líquido que es producto de un valor de masa y de un volumen desplazado, esto implica el uso de métodos tradicionales de calibración que pueden ser optimizados con la introducción de herramientas que semiautomaticen el proceso de ajuste del menisco, lo que mejora la repetibilidad del proceso por un aumento en la estabilidad del proceso, aumentando la exactitud de los resultados y disminuyendo la incertidumbre estimada en el proceso de calibración.
2.2.3 Factores de influencia en la incertidumbre

a. Incertidumbre por la repetibilidad (desviación estándar) de los datos:

$u_{\text {repet }}=\frac{S\left(\Delta \rho_{x i}\right)}{\sqrt{n}}$

En donde:

$n \quad$ número de repeticiones realizadas

$\rho_{x i} \quad$ densidad de la incógnita

$S$ desviación estándar del proceso de medición y se obtiene de la siguiente forma:

$S\left(\Delta \rho_{x i}\right)=\sqrt{\frac{1}{n-I}\left(\sum_{i=1}^{n}\left(\Delta \rho_{i}-\overline{\Delta \rho}\right)^{2}\right)}$

b. Incertidumbre por la resolución del hidrómetro

$u_{\text {resol }}=\frac{d}{m \sqrt{3}}$

En donde:

d mínima división de escala del

$m$ hidrómetro dividir la mínima división de escala

c. Incertidumbre por la masa del hidrómetro en el aire

En donde:

$u_{m^{c}{ }_{p e s a s}} \quad$ incertidumbre de las pesas patrón usadas

$c_{m_{\text {pesasl }}^{c}} \quad$ coeficiente de sensibilidad de las pesas patrón usadas

$u_{\rho_{\text {airel }}}$ 
$u\left(m_{\text {aire }}\right)=\sqrt{\left(c_{m_{\text {pesas } 1}^{c}} u_{m_{\text {pesas } 1}^{c}}\right)^{2}+\left(c_{\rho_{\text {aire } 1}} u_{\rho_{\text {aire } 1}}\right)^{2}+\left(c_{\rho_{\text {pesas }}} u_{\rho_{\text {pesas }}}\right)^{2}+\left(c_{\Delta m 1} u_{\Delta m 1}\right)^{2}}$

$$
\begin{aligned}
& c_{\rho_{\text {airel }}} \\
& u_{\rho_{\text {pesas }}} \\
& c_{\rho_{\text {pesas }}} \\
& u_{\Delta m l} \\
& c_{\Delta m 1}
\end{aligned}
$$

aire durante la medición de la masa coeficiente de sensibilidad por la densidad del aire durante la medición de la masa incertidumbre por la densidad de las pesas patrón usadas coeficiente de sensibilidad por la densidad de las pesas patrón usadas incertidumbre por la diferencia en la lectura de la masa coeficiente de sensibilidad por la diferencia en la lectura de la masa

d. Incertidumbre por la masa del hidrómetro en el punto a calibrar (sumergido)

En donde:

$u_{m_{\text {pesas } 2}^{c}}$
$c_{m^{c}{ }_{\text {pesas } 2}}$
$u_{\rho_{\text {aire } 2}}$
$c_{\rho_{\text {aire } 2}}$
$u_{\rho_{\text {pesas }}}$
$c_{\rho_{\text {pesas }}}$
$u_{\Delta m 2}$
$c_{\Delta m 2}$

incertidumbre de las pesas patrón usadas coeficiente de sensibilidad de las pesas patrón usadas

incertidumbre por la densidad del aire durante la medición de la masa coeficiente de sensibilidad por la densidad del aire durante la medición de la masa

incertidumbre por la densidad de las pesas patrón usadas coeficiente de sensibilidad por la densidad de las pesas patrón usadas incertidumbre por la diferencia en la lectura de la masa coeficiente de sensibilidad por la diferencia en la lectura de la masa e. Incertidumbre por la densidad del líquido

La densidad del líquido, en este caso agua ultra pura, se determina con la siguiente ecuación:

$\rho_{w}=a_{5} \times\left[1-\frac{\left(t+a_{1}\right)^{2}\left(t+a_{2}\right)}{a_{3}\left(t+a_{4}\right)}\right] \times f_{c}+C_{g d}$

En donde:

$\begin{array}{ll}a_{1} & -3,983035^{\circ} \mathrm{C} \pm 0,00067{ }^{\circ} \mathrm{C} \\ a_{2} & 301,797{ }^{\circ} \mathrm{C} \\ a_{3} & 522528,9{ }^{\circ} \mathrm{C}^{2} \\ a_{4} & 69,34881{ }^{\circ} \mathrm{C} \\ a_{5} & 999,972 \mathrm{~kg} \mathrm{~m}^{-3} \pm 0,00084 \mathrm{~kg} \mathrm{~m}^{-3} \\ t & \text { temperatura en }{ }^{\circ} \mathrm{C} \\ f_{c} & \text { factor de compresibilidad } \\ C_{g d} & \text { corrección por gases disueltos }\end{array}$

La incertidumbre por la densidad del líquido (agua ultra pura), se determina con la siguiente ecuación:

En donde:

$u_{\text {temp }} \quad$ incertidumbre por la temperatura del agua

$c_{\text {temp }}$ coeficiente de sensibilidad por la temperatura del agua

$u_{\text {pres }} \quad$ incertidumbre por la presión atmosférica

$c_{\text {pres }} \quad$ coeficiente de sensibilidad por la presión atmosférica

$u_{a_{l}} \quad$ incertidumbre por el valor de $a_{l}$

$c_{a_{l}} \quad$ coeficiente de sensibilidad por el valor de $a_{1}$

$u_{a_{5}} \quad$ incertidumbre por el valor de $a_{5}$

$$
\begin{aligned}
& u\left(m_{\text {atre }}\right)=\sqrt{\left(c_{m_{\text {pesas } 2}^{c}} u_{m_{\text {pessas } 2}^{c}}\right)^{2}+\left(c_{\rho_{\text {aire } 2}} u_{\rho_{\text {atire } 2}}\right)^{2}+\left(c_{\rho_{\text {pesas }}} u_{\rho_{\text {pesas }}}\right)^{2}+\left(c_{\Delta m_{2}} u_{\Delta m_{2}}\right)^{2}} \\
& u_{\rho_{I}}=\sqrt{\left(c_{\text {temp }} u_{\text {temp }}\right)^{2}+\left(c_{\text {pres }} u_{\text {pres }}\right)^{2}+\left(c_{a_{1}} u_{a_{1}}\right)^{2}+\left(c_{a_{5}} u_{a_{5}}\right)^{2}+\left(c_{\text {produc }} u_{\text {produc }}\right)^{2}}
\end{aligned}
$$


$c_{a_{5}} \quad$ coeficiente de sensibilidad por el valor de $\mathrm{a}_{5}$

$u_{\text {produc }} \quad$ incertidumbre por la producción del agua ultra pura

$c_{\text {produc }} \quad$ coeficiente de sensibilidad por la producción del agua ultra pura

f. Incertidumbre por la densidad del aire

El cálculo de la densidad del aire se realiza con la fórmula de sugerida por el BIPM (CIPM), revisión de 2007.

$\rho_{a}=\frac{p M_{a}}{Z R T}\left[1-x_{v}\left(1-\frac{M_{v}}{M_{a}}\right)\right]$

En donde:

$\rho_{a} \quad$ Densidad del aire

$p \quad$ Presión atmosférica

$M_{a} \quad$ Masa molar de aire seco

$M_{a} \quad 28,96546 \times 10^{-3} \mathrm{~kg} / \mathrm{mol}$

$M_{v} \quad$ Masa molar de agua

$M_{v} \quad 18,01528 \times 10^{-3} \mathrm{~kg} / \mathrm{mol}$

$Z \quad$ Factor de compresibilidad

$R \quad$ Constante de los gases

$R \quad 8,314510 \mathrm{~J} /(\mathrm{mol} \cdot \mathrm{K})$

$T \quad$ Temperatura absoluta

$T(K) \quad 273,15+t$

$x_{v} \quad$ Fracción molar de vapor de agua.

$t$ Temperatura por la pesada en el aire

$x_{v}=h f_{(p, t)} \frac{p_{s v}(t)}{p}$

En donde:

$h \quad$ humedad relativa

$p \quad$ presión atmosférica

$p_{s v}(t) \quad$ presión de vapor saturado en el aire

$f \quad$ factor de fugacidad
La incertidumbre por la densidad del aire se determina con la siguiente ecuación:

En donde:

$u_{\text {pres }}$ incertidumbre por la presión atmosférica

$c_{\text {pres }}$ coeficiente de sensibilidad por la incertidumbre de la presión atmosférica

$u_{\text {temp }} \quad$ Incertidumbre por la temperatura del aire

$c_{\text {temp }}$ coeficiente de sensibilidad por la incertidumbre de la temperatura del aire

$\begin{array}{ll}u_{\text {hum }} & \text { incertidumbre por la humedad relativa } \\ c_{\text {hum }} & \text { coeficiente de sensibilidad por la }\end{array}$ incertidumbre de la humedad relativa

$u_{R} \quad$ incertidumbre por la constante internacional de los gases

$c_{R} \quad$ coeficiente de sensibilidad por la incertidumbre de la constante internacional de los gases

$u_{\text {есиас }}$ incertidumbre por la ecuación de cálculo de la densidad del aire, que tiene un valor de $\left(\left(1 \times 10^{-4}\right) \cdot \rho_{a}\right)$, para el ámbito recomendado de uso: 600 $\mathrm{hPa} \leq \mathrm{p} \leq 1100 \mathrm{hPa}$ y $15^{\circ} \mathrm{C} \leq \mathrm{t} \leq 27^{\circ} \mathrm{C}$ coeficiente de sensibilidad por la incertidumbre de la ecuación del cálculo de la densidad

g. Incertidumbre por el diámetro del vástago del hidrómetro

$u_{D}=\sqrt{u_{D 1}^{2}+u_{D 2}^{2}+u_{D 3}^{2}}$

En donde:

$u_{D I} \quad$ incertidumbre del equipo usado para la medición del diámetro

$u_{D 2} \quad$ incertidumbre por la resolución del equipo usado para la medición del diámetro

$u_{\rho, \text { aire }}=\sqrt{\left(c_{\text {pres }} u_{\text {pres }}\right)^{2}+\left(c_{\text {temp }} u_{\text {temp }}\right)^{2}+\left(c_{\text {hum }} u_{\text {hum }}\right)^{2}+\left(c_{R} u_{R}\right)^{2}+\left(c_{\text {eсиас }} u_{\text {eсиас }}\right)^{2}}$ 
$u_{D 3} \quad$ incertidumbre por la diferencia en las mediciones del diámetro

h. Incertidumbre por la temperatura del aire

$u_{\text {t@aire }}=\sqrt{u_{\text {t@airel }}^{2}+u_{\text {t@aire2 }}^{2}+u_{\text {t@aire3 }}^{2}}$

En donde:

$u_{\text {@@airel }} \quad$ incertidumbre del equipo usado para la medición de la temperatura del aire incertidumbre por la resolución del equipo usado para la medición de la temperatura del aire

$u_{\text {t@aire2 }}$ $u_{t @ a i r e 3}$

incertidumbre por la diferencia en las mediciones de la temperatura del aire

En donde:

$u_{t @ a g u a l}$

$u_{t @ a g u a 2}$ incertidumbre del equipo usado para la medición de la temperatura del agua incertidumbre por la resolución del equipo usado para la medición de la temperatura del agua

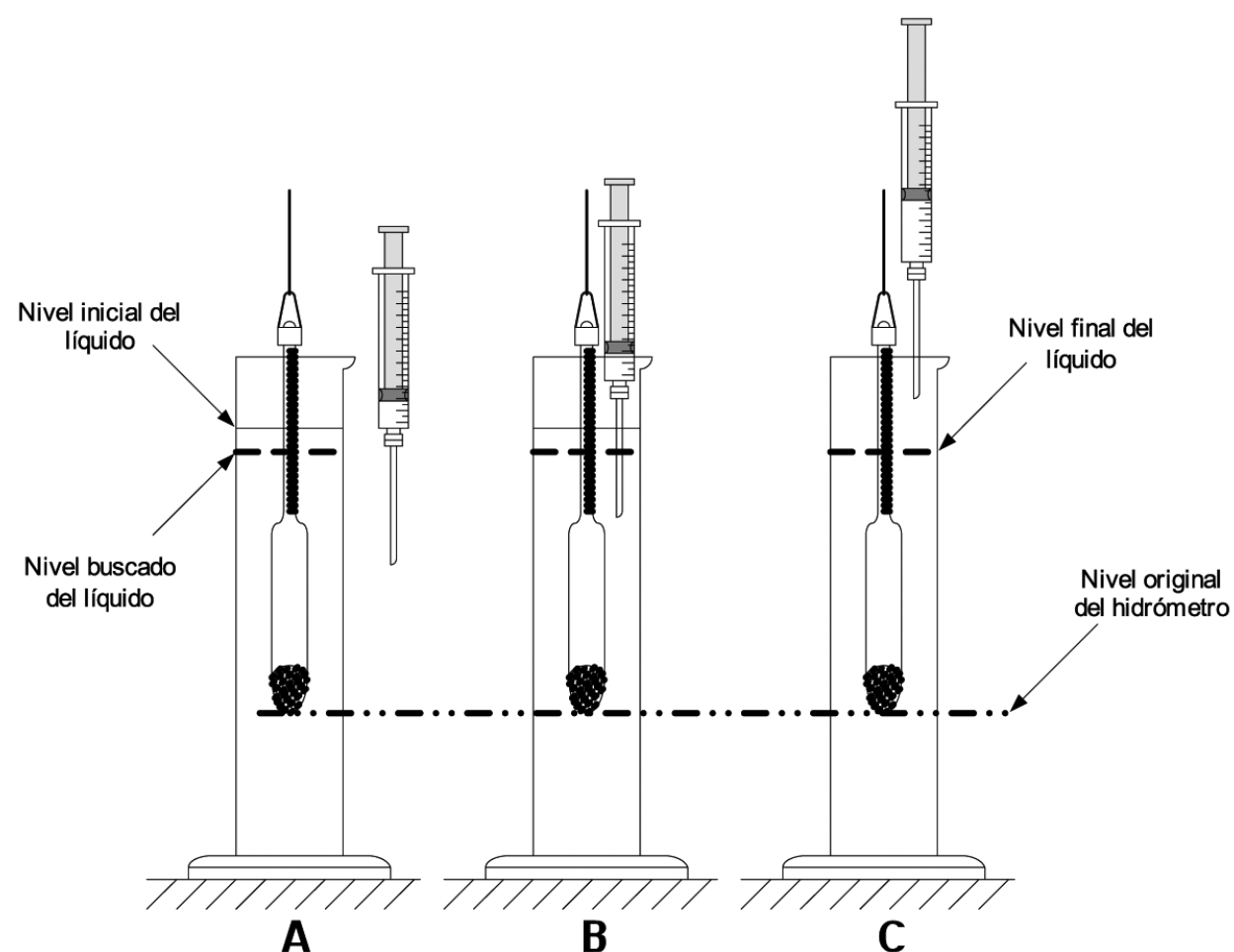

Figura 2. Ajuste del nivel del líquido en el recipiente con el uso de una jeringuilla.

Fuente: (El autor). 
$u_{t @ a g u а 3 ~}$ incertidumbre por la diferencia en las mediciones de la temperatura del agua

j. Incertidumbre por el coeficiente cúbico de dilatación térmica del hidrómetro

El coeficiente cúbico de dilatación térmica " $\beta$ " de los hidrómetros se puede obtener de la literatura. Por ejemplo la Norma ISO 4787, 1984 (p. 9, Tabla 5) y algunas referencias técnicas brindadas por los fabricantes (Catálogo General Brand 400 , información técnica, p. 12) permiten determinar este valor de acuerdo con la composición del vidrio: sódico cálcico es de $25 \times 10^{-6} \mathrm{C}^{-1}$, boro silicato es de $10 \times 10^{-6}{ }^{\circ} \mathrm{C}^{-1}$, con una incertidumbre estándar de $1,2 \times 10^{-6}{ }^{\circ} \mathrm{C}^{-1}$, considerando esta incertidumbre como una distribución de probabilidad rectangular se obtiene:

$$
u_{\bar{\beta}} \quad \frac{u_{\beta}}{\sqrt{3}}=\frac{12 \times 10^{-6}}{2 \sqrt{3}}
$$

k. Incertidumbre por la aceleración local de la gravedad

La aceleración local de la gravedad es obtenida del estudio de Leandro (1998), medición de la gravedad observada en ONNUM y Escuela de Física / UCR, Reporte Técnico de la Oficina de Geofísica Aplicada del ICE, con un valor de $\left(9,77906254 \pm 48 \times 10^{-8}\right) \mathrm{m} / \mathrm{s}^{2}$.

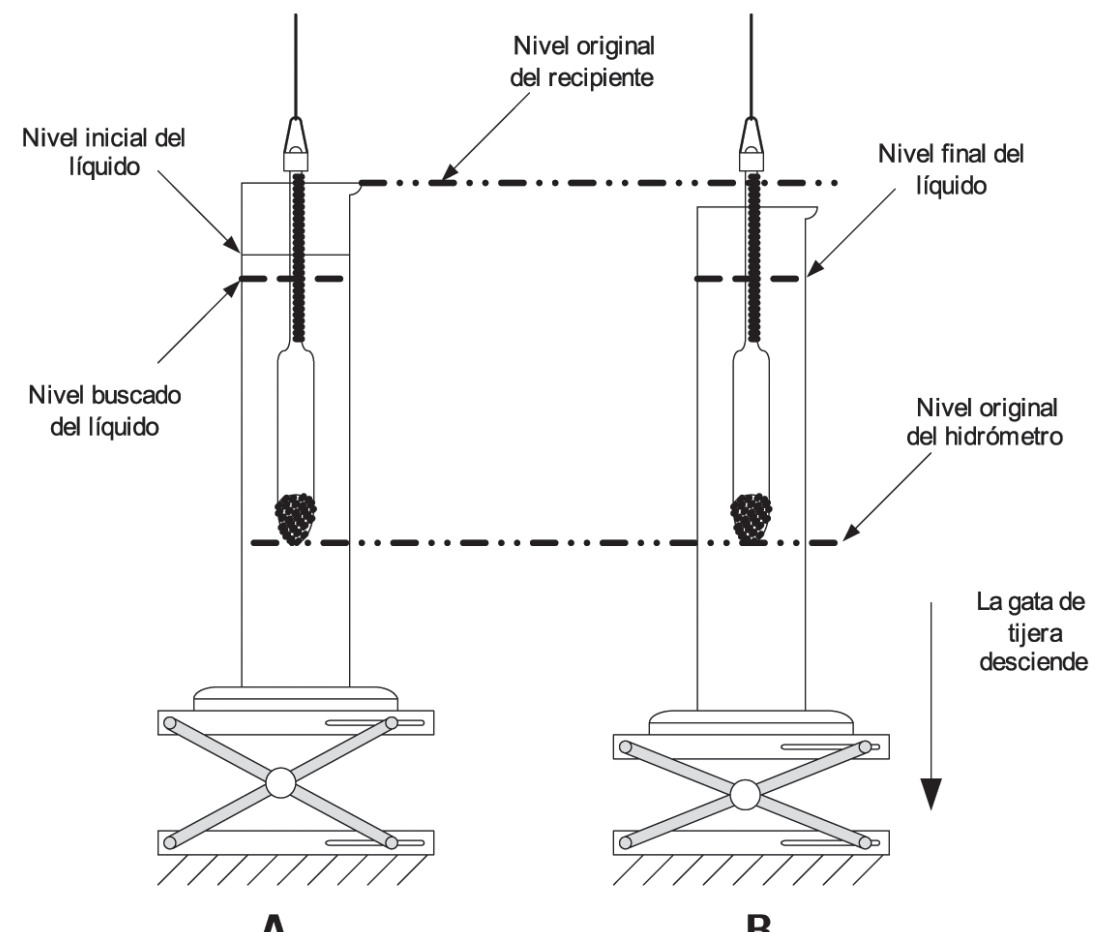

Figura 3. Ajuste del nivel del líquido en el recipiente con el uso de una gata de tijera.

Fuente: (El autor). 


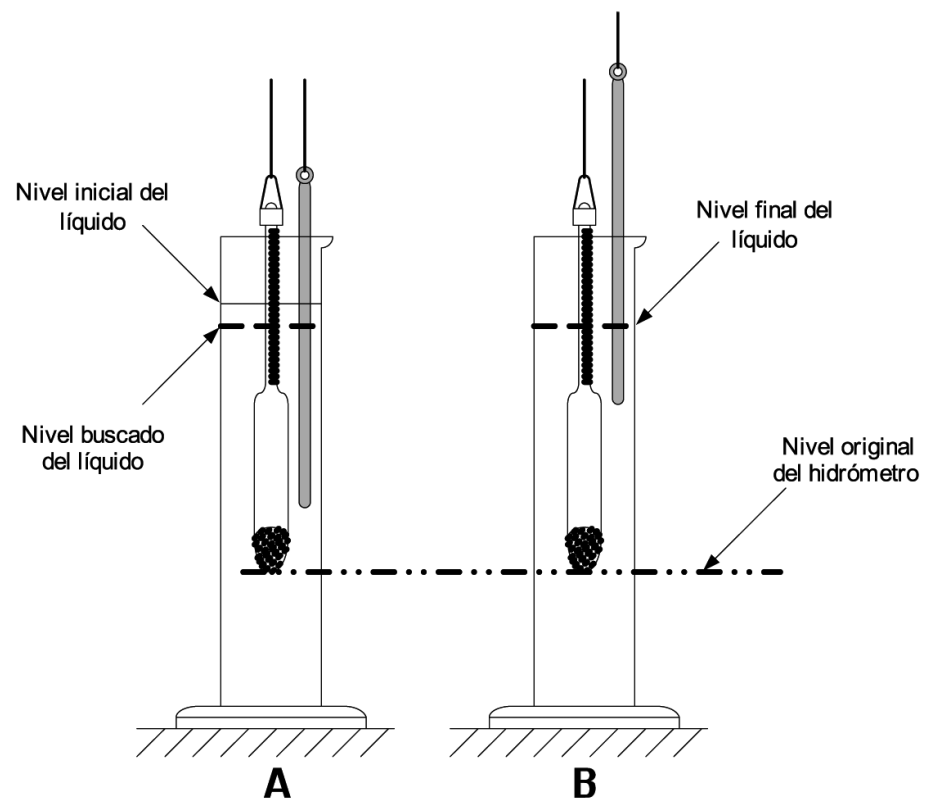

Figura 4. Ajuste del nivel del líquido en el recipiente con el uso de una varilla de vidrio.

Fuente: (El autor).

1. Incertidumbre por la tensión superficial del líquido patrón

Se determina la ecuación de la relación de tensión superficial y la densidad o temperatura y se calcula el coeficiente de sensibilidad (pendiente cerca del punto donde se encuentra la densidad medida) en los cuadros utilizados y se considera una distribución rectangular, ver Cuadro 2 de la sección 3.

\subsection{Ajuste del menisco}

Existen tres formas de ajustar el menisco formado entre el material del cuello y el líquido de medición en el proceso de calibración de hidrómetros, en todas ellas se desplaza el nivel del líquido hasta hacerlo coincidir con la escala del hidrómetro en la marca de calibración. a. Agregar o quitar líquido

El hidrómetro se coloca dentro del recipiente que contiene el líquido de medición y se deja suspendido (flotando) hasta alcanzar el equilibrio (ver Figura 2A). Para ajustar al nivel de medición adecuado se utiliza un gotero o una jeringuilla para introducir o sacar líquido del recipiente en donde se encuentra el hidrómetro hasta alcanzar la marca deseada, tal y como lo muestran las Figuras 2B y $2 \mathrm{C}$.

\section{b. Ajuste externo de nivel}

Se coloca y asegura una gata de tijera en el lugar de calibración; sobre la misma se coloca el recipiente que va a contener el líquido de medición y el hidrómetro. El hidrómetro se coloca dentro del recipiente que contiene el líquido de medición y se deja suspendido 
(flotando) hasta alcanzar el equilibrio (ver Figura 3A). Para ajustar al nivel de medición adecuado se mueve la plataforma de la gata de tijera subiendo o bajando el nivel del recipiente que contiene el líquido de medición y se iguala el nivel a la marca de calibración deseada, tal y como lo muestra la Figura 3B.

\section{c. Ajuste con el uso de una varilla de vidrio}

Dentro del recipiente que contiene el líquido de medición se coloca el hidrómetro y se deja suspendido (flotando) hasta alcanzar el equilibrio, al mismo tiempo se introduce una varilla de vidrio que cuelga suspendida dentro de este mismo recipiente que debe estabilizar junto con el hidrómetro. La varilla de vidrio tiene la función de aumentar el nivel del líquido de medición ya que al ser introducida dentro del recipiente aumenta el volumen del líquido de acuerdo con la cantidad de varilla que se encuentre sumergida en el mismo (ver Figura 4A). Para ajustar al nivel de medición adecuado se utiliza una varilla delgada de vidrio sólido, la cual se introduce o se saca del recipiente en el cual están contenidos el líquido y el hidrómetro, aumentando o disminuyendo el volumen del líquido de medición hasta ajustar a la marca deseada, tal y como lo muestra la Figura 4B.

Una de las condiciones o requisitos más importantes que deben ser considerados en el proceso de calibración de hidrómetros es la estabilidad del líquido de medición. La estabilidad del líquido de medición consiste en la eliminación de las vibraciones que pueden ser transmitidas a través del mismo hasta el hidrómetro y que por ende, pueden ser transmitidas hacia la balanza que está siendo utilizada en el proceso de calibración. El método adecuado de ajuste del nivel de lectura (o menisco) debe ser elegido con base en el nivel de estabilidad adecuado, este método de ajuste influye en el nivel de exactitud deseado en la medición, ya que cuanto más estable y rápido sea el proceso de ajuste habrá un menor aumento en la temperatura del líquido de medición y así, una menor variación en las propiedades de este fluido.

Para lograr un nivel de control y estabilidad óptimos es posible mejorar el método de ajuste del nivel de lectura (o menisco) usando un motor que de forma manual o automatizada controle el ingreso y la salida de la barra de vidrio. Este control automatizado del proceso de calibración de hidrómetros puede ser realizado con la implementación de un sistema semi-automatizado de ajuste y de adquisición de datos.

Este sistema semi-automatizado de ajuste y de adquisición de datos se puede construir con elementos de uso común y con una aplicación realizada en LabVIEW ${ }^{\circledR}$ el cual es un software de tipo gráfico creado para ser usado en aplicaciones de ingeniería que cuenta con
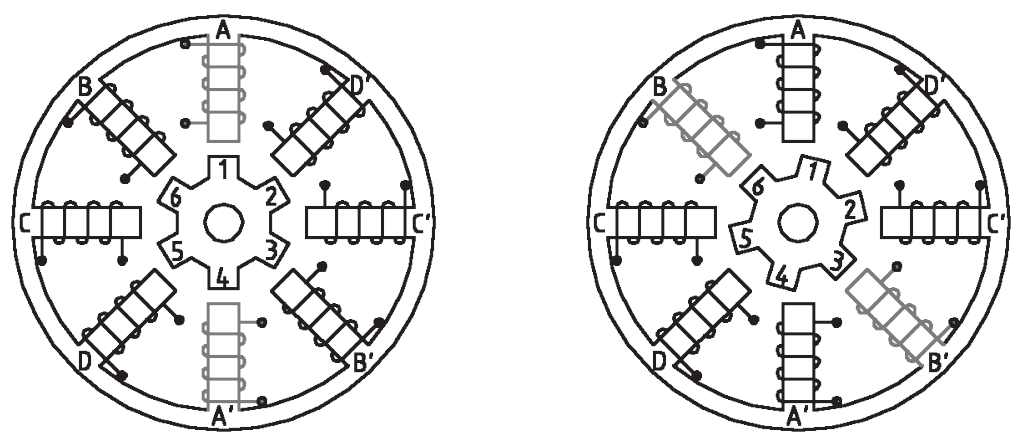

Figura 5. Secuencia de fase completa de un paso de un motor unipolar.

Fuente: (El autor). 
módulos prediseñados para labores de control. En el proceso de calibración de hidrómetros con el uso del Método de Cuckow algunos de los módulos de este software van a ser usados para controlar un motor e integrar varios equipos en una sola aplicación, como la lectura de datos colectados desde la balanza y las indicaciones de temperatura, presión y humedad de los equipos para la medición de condiciones ambientales y datos que son críticos de controlar y registrar al realizar la calibración.

\subsection{Elementos que integran el sistema de control}

\section{a. Motor Paso a Paso}

Un motor paso a paso es un artefacto destinado a convertir energía eléctrica en energía rotacional, se componen de un estator formado por un conjunto de bobinas y un rotor sólido en forma de estrella, fabricado con una sustancia paramagnética o con un imán permanente. En los motores paso a paso se induce una corriente eléctrica a las bobinas del estator para producir un campo magnético, dentro de este campo magnético se encuentra el rotor el cual por la naturaleza del material con el que fue construido será atraído cada vez que una bobina es energizada, esto produce el movimiento rotacional del eje.

Un motor paso a paso tiene la característica particular de poder ser controlado de manera que su velocidad y posicionamiento se produzca en un paso a la vez, cada paso será un ángulo de giro que fácilmente puede ser determinado, esto permite contar con un motor de movimientos muy precisos y controlados.

El motor paso a paso usado en este sistema de control es de tipo unipolar extraído de una impresora fuera de funcionamiento; cuenta con

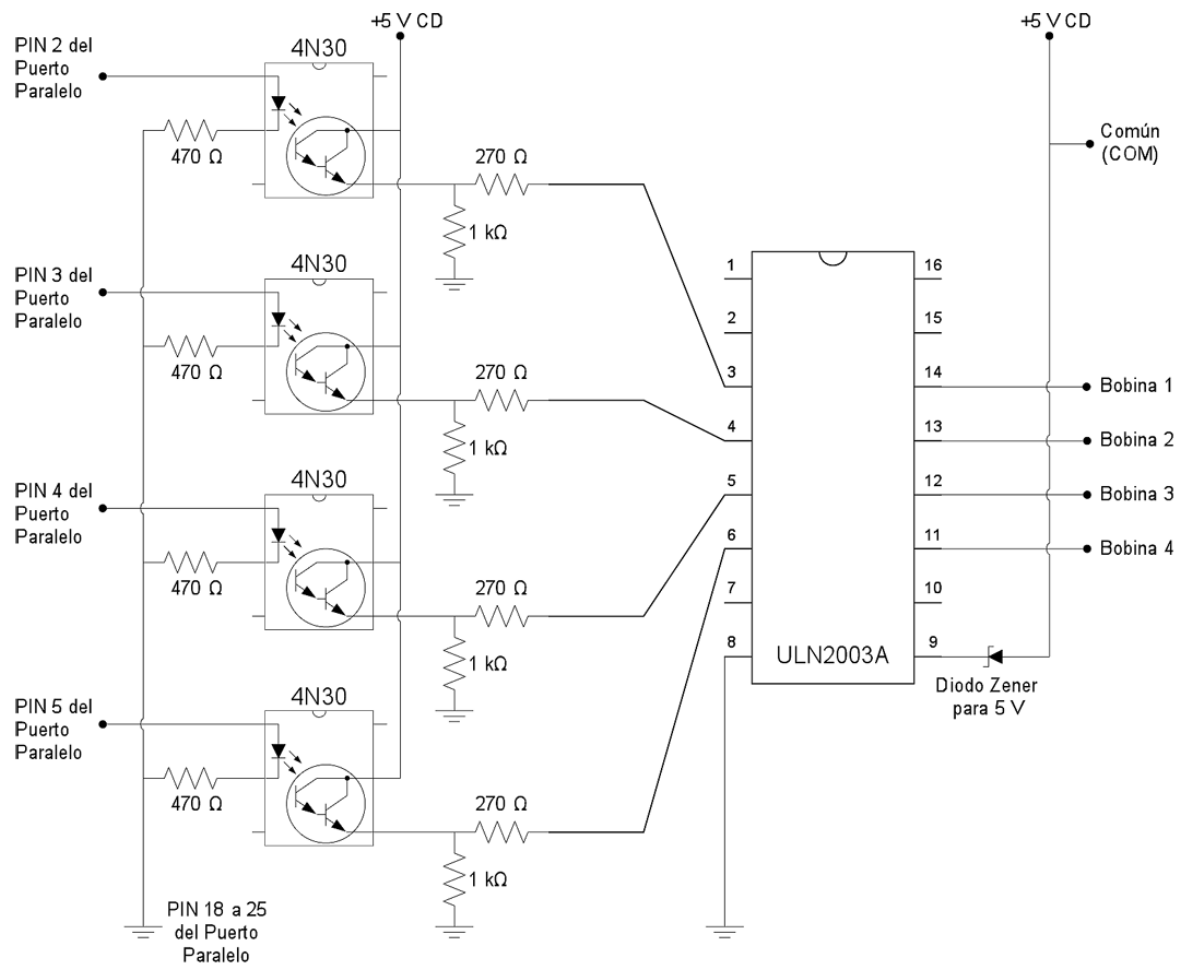

Figura 6. Circuito de control.

Fuente: (El autor). 
un estator cilíndrico con forma de estrella, con ocho dientes alrededor de cada uno de los cuales se arrolla una bobina de alambre conductor conectada en serie; las bobinas están ubicadas una frente a la otra, lo que forma cuatro fases de dos bobinas conectadas en cada fase. Los motores paso a paso de tipo unipolar pueden presentar entre cinco y ocho cables, sin embargo, cuatro corresponden a cada fase y el resto serán cables comunes por donde se suministra el voltaje de alimentación, el motor seleccionado presenta cinco cables de los cuales uno es el común.

El funcionamiento y la disposición de la secuencia de fase completa considerada como adecuada para el caso en estudio se explica a continuación (ampliar en Harries, 2006).
La Figura 5 presenta el diagrama de un motor de cuatro fases de tipo unipolar, similar al motor usado en esta aplicación. Cada fase se identifica con las letras A, B, C, D, cada una de ellas se compone de dos bobinas identificadas como A, A', B, B', C, C', D y D', las cuales forman parejas tal y como se muestra en la Figura, por ejemplo A y A', que se ubican una frente a la otra o lo que sería en posición opuesta y en serie, pero devanadas al revés, de tal manera que al energizar cada fase se energice cada par de bobinas al mismo tiempo y así cada bobina mostrará un polo magnético diferente. El rotor de imán permanente tiende a alinearse de tal forma que el mayor número de líneas de fuerza lo atraviesen. En la Figura 5 esto se puede ver cuando la fase

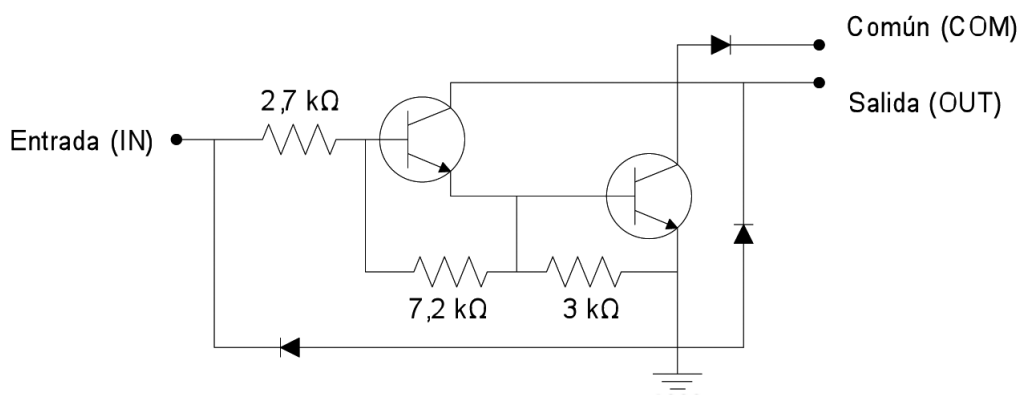

Figura 7. Circuito interno del ULN2003.

Fuente: (Autor, con base en la hoja de datos del circuito integrado ULN2003).

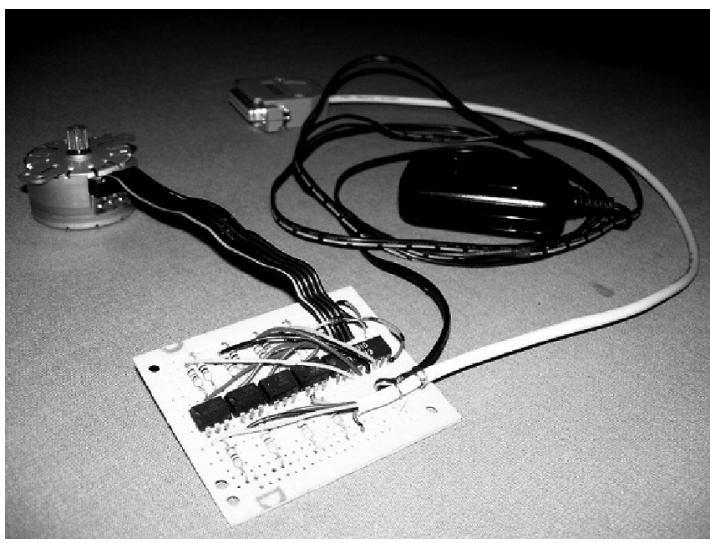

Figura 8. Circuito de control, motor paso a paso, cables de conexión y tren de engranajes.

Fuente: (El autor). 
A se energiza, en este caso los dientes 1 y 4 se alinean con las bobinas A y A', respectivamente. Para mover el rotor hacia el siguiente paso se desenergiza la fase A y de inmediato se energiza la fase B. Al energizar la fase B, las bobinas B y B' se energizan y el rotor se mueve de tal forma que los dientes más cercanos queden alineados con las bobinas energizadas, en este caso el diente 6 con la bobina B y el diente 3 con la bobina B'. Las cuatro fases de este motor se cumplen al energizar cada fase una a la vez o en conjunto sincronizado.

Este proceso de energizar las bobinas una a una y en una secuencia establecida es el que permite que la barra de vidrio, utilizada para ajustar al nivel de medición adecuado, tal y como muestra la Figura 4, ascienda y descienda de forma controlada. LabVIEW ${ }^{\circledR}$ realiza este proceso por medio del puerto paralelo de la PC y el uso de un circuito de control que envía a cada bobina un pulso de voltaje conocido.

\section{b. Circuito de control}

En un sistema automatizado la señal de mando es generada desde la PC o desde un microcontrolador, esta señal de mando puede ser cualquier instrucción conocida, cuando esto sucede el circuito de control puede recibir

Cuadro 1. Asignación de valores para la determinación del giro del motor.

\begin{tabular}{cccc}
\multicolumn{3}{c}{ Giro a la derecha } \\
Registro & $\begin{array}{c}\text { Pin del puerto } \\
\text { paralelo }\end{array}$ & $\begin{array}{c}\text { Identificación } \\
\text { decimal }\end{array}$ & $\begin{array}{c}\text { Identificación Binaria } \\
\text { (bits utilizados del registro 888) }\end{array}$ \\
888 & 2 & 1 & 00000001 \\
888 & 3 & 2 & 00000010 \\
888 & 4 & 4 & 00000100 \\
888 & 5 & 8 & 00001000 \\
& & &
\end{tabular}

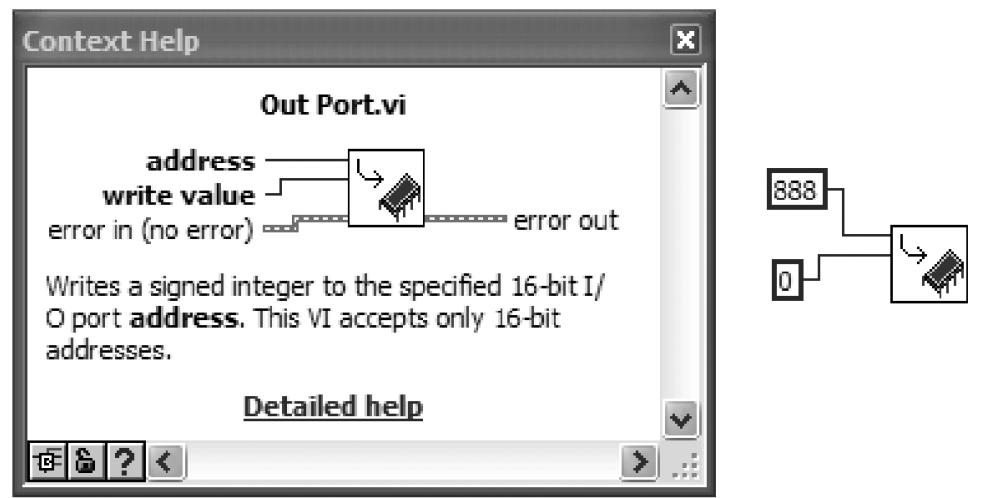

Figura 9. Apertura del puerto paralelo con LabVIEW ${ }^{\circledR}$. Fuente: (El autor). 
esta señal, interpretarla y realizar una acción determinada. Para el proceso de calibración de hidrómetros con el uso del Método de Cuckow la acción buscada es hacer que el eje de un motor gire en sentido horario y anti-horario, este eje estará conectado a un sistema mecánico de engranajes que se moverá para producir la acción previamente planificada de ascenso y descenso de la barra de vidrio. Las características de los elementos discretos que componen el circuito, sus propiedades y recomendaciones pueden encontrarse en Sinclair y Duton (2007).

El circuito de control consta de varias partes, la primera parte está formada por cuatro opto- acopladores 4N30 de salida tipo Darlington, los cuales permiten separar el área de mando, que en este caso es una PC, del resto del circuito de control. Cada pin del puerto paralelo será energizado de forma ordenada por medio de un programa diseñado para este fin, el fotoemisor excitará al foto-transistor para producir una salida normal de $5 \mathrm{~V}$. La segunda parte del circuito de control está formada por un circuito integrado comercial con arreglo interno de transistores tipo Darlington, el ULN2003; para este integrado la salida de voltaje que entrega el foto-transistor del $4 \mathrm{~N} 30$ es suficiente para producir $5 \mathrm{~V}$ de salida con hasta $500 \mathrm{~mA}$,

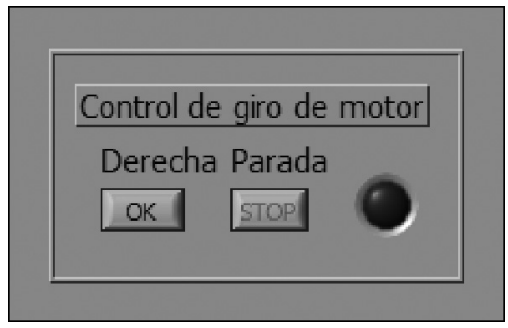

Figura 10. Panel frontal, secuencia de giro, motor paso a paso con LabVIEW ${ }^{\circledR}$.

Fuente: (El autor).

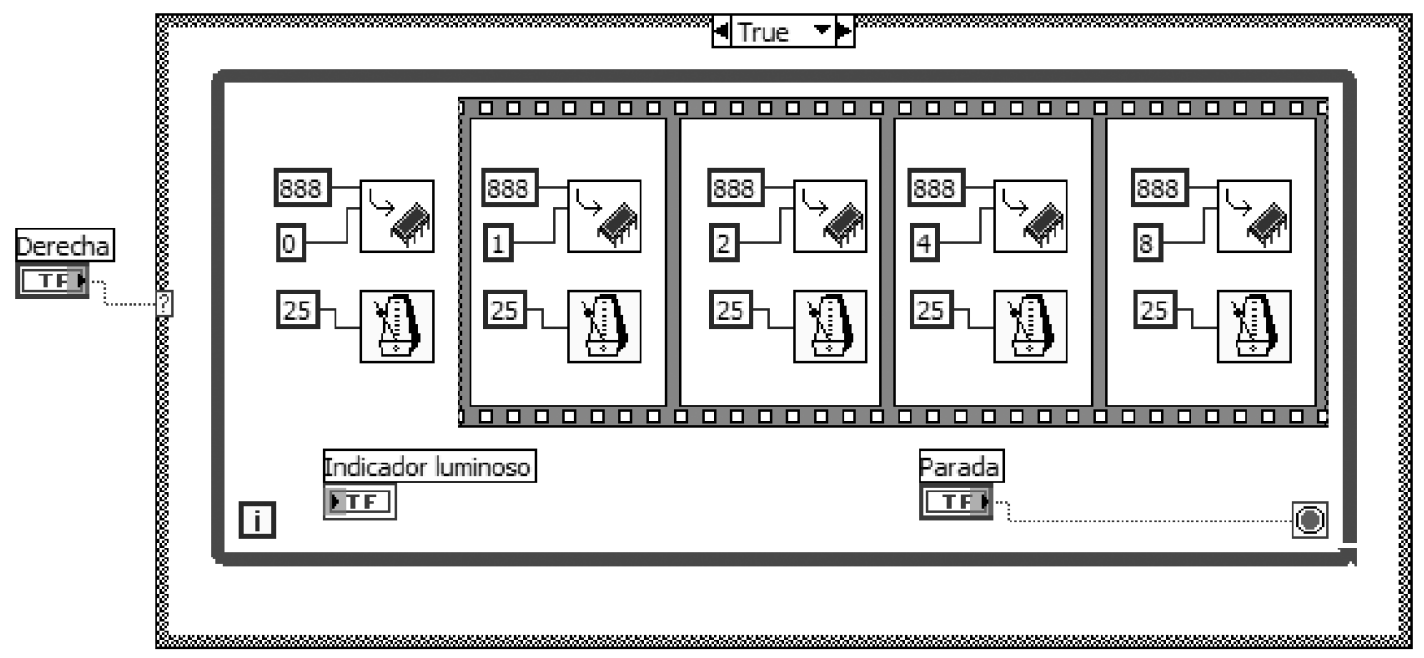

Figura 11. Secuencia de giro para un motor paso a paso con LabVIEW ${ }^{\circledR}$ 
lo cual es también suficiente para el manejo del motor con el cual se desarrolló el sistema de control para el proceso de calibración de hidrómetros.

El ULN2003 presenta el siguiente circuito interno.

El ULN2003 tiene siete entradas de activación y siete salidas de control, la distribución de los elementos integrados presenta dos diodos internos de protección; por otro lado, al armar el circuito es altamente recomendable colocar entre la entrada de voltaje y el pin 9 del ULN2003 un diodo Zener de $5 \mathrm{~V}$, si se trabaja con este voltaje, el cual absorbe cualquier fuerza electromotriz generada por el colapso del campo magnético del motor cuando las bobinas son apagadas. La secuencia de encendido de las bobinas del motor paso a paso debe ser definida de antemano y serán conectadas según se muestra en la Figura 6; el alambre común, de entrada de voltaje del motor será conectado a la entrada de $5 \mathrm{~V}$.

La conexión del circuito y el motor paso a paso quedó de la siguiente forma.

\section{c. Programa de control en LabVIEW ${ }^{\circledR}$}

El software LabVIEW ${ }^{\circledR}$, de National Instruments permite usar el puerto paralelo como medio de comunicación, para esta aplicación el puerto paralelo es usado como medio de envío de datos hacia el circuito de control (ampliar en Bitter, Mohiunddin \& Nawrocki, 2007; Sumathi \& Surekha, 2007).

En el puerto paralelo los pines que pueden ser usados para el envío de datos son especialmente los del Registro de Datos, aquellos que van desde el pin 2 hasta el pin 9, por lo tanto se cuenta con ocho salidas TTL las cuales permiten controlar al menos ocho bobinas. Para el desarrollo del sistema de control para el proceso de calibración de hidrómetros sólo se necesitaron cuatro pines, específicamente del 2 al 5 , tal y como se observa en el circuito de la Figura 7.

Para poder acceder al puerto paralelo es necesario conocer el nombre o número de identificación asignado por Windows, o cualquier otro sistema operativo al Registro de Datos, posteriormente es necesario asignar el número de pin que se desea acceder. Por lo general el registro de datos se identifica con 888 en decimal y 378 en hexadecimal, el pin accedido dependerá de los bits utilizados y disponibles en cada registro, para el caso del Registro de Datos se dispone de 8 bits. La forma en la que se determinan las direcciones, tomando en cuenta cada bit se hace de la siguiente forma.

Acceder al puerto paralelo y energizar los pines 2, 3, 4 y 5 se realizaría con el Out Port. VI, de la siguiente manera.

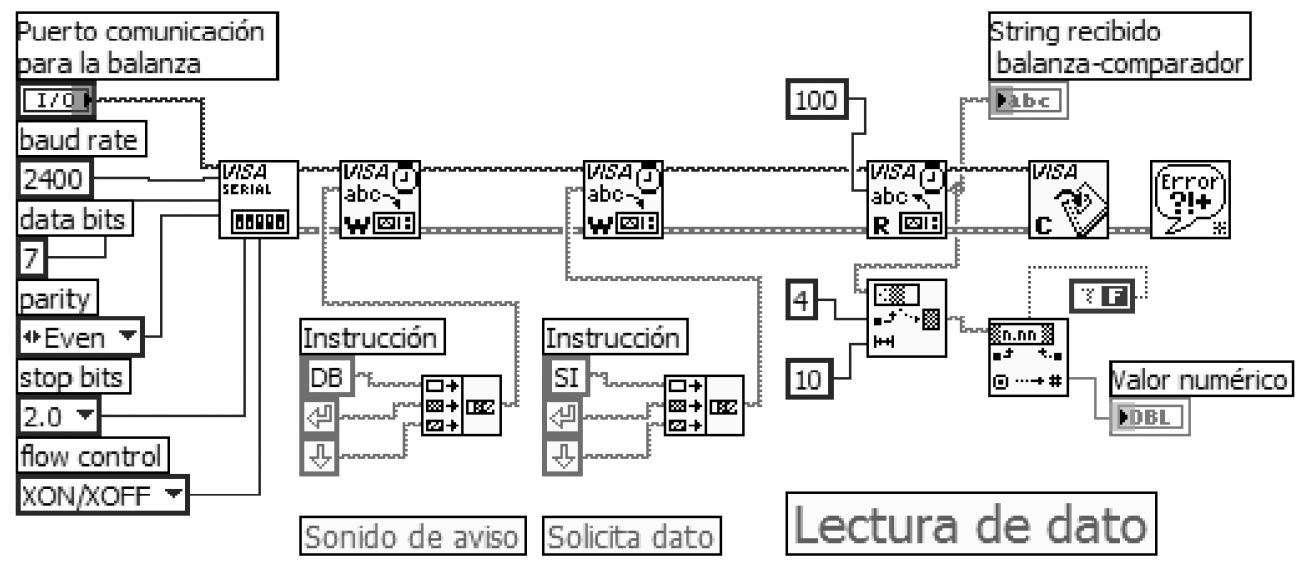

Figura12. Subrutina de comunicación vía puerto serial con LabVIEW ${ }^{\circledR}$. 
La Figura 9 muestra el icono que carga la subrutina de apertura del puerto paralelo; a esta subrutina es necesario indicarle la dirección del registro del puerto paralelo que se va a usar y el pin que será energizado; en el caso de la Figura 9 el valor 888 será la dirección e indicará el registro de datos mientras tanto, el valor de cero indica que no se energiza ningún pin, por el contrario, esta orden des-energiza todos los pines del registro de datos.

Una vez que se conoce la forma en la cual se accede al puerto paralelo, es posible planificar una secuencia que energice de forma adecuada los pines necesarios del registro de datos del puerto paralelo para hacer un giro completo del eje del motor paso a paso. Una rotación del eje del motor hacia la derecha, por ejemplo, estará controlada por una subrutina que consta de dos partes, un panel frontal de control que es el área en la cual el usuario activa, condiciona o apaga el programa, y una secuencia de acciones activada por el panel frontal de control. La Figura 10 muestra el diseño de un posible panel de control, este cuenta con únicamente dos botones, el botón de dirección enciende la secuencia de giro hacia la derecha, el botón de parada apaga el giro, por otro lado un indicador lumínico permite saber cuándo se realiza el giro ya que se enciende al ocurrir el mismo.
Para realizar la secuencia de giro a la derecha y relacionando este giro con el panel de la Figura 10, se construyó la secuencia de la Figura 11 en la cual cada pin será energizado, uno a la vez, cuando el usuario active el ciclo condicional al activar el botón de giro a la derecha. El ciclo de secuencia dentro de un ciclo while se repetirá hasta que el usuario decida detener el proceso, de esa forma cada bobina será energizada para mantener la rotación, esto produce el giro necesario para que la barra de vidrio de ajuste del menisco suba o descienda dentro del recipiente. La frecuencia con la cual ocurre la rotación está determinada por la frecuencia con la cual el impulso enviado desde el puerto paralelo es transmitido a cada bobina, la Figura 8 muestra un temporizador programado para cada $25 \mathrm{~ms}$, este temporizador determina la frecuencia con la que las bobinas se energizan y de hecho la velocidad de rotación, esta frecuencia es característica de cada motor y su construcción, sin embargo, si se agrega un tren de engranajes, como sucede en este caso, es recomendable hacer pruebas para regular la frecuencia de forma adecuada. La determinación de la frecuencia de giro es uno de los puntos críticos en la implementación del sistema, la estabilidad del proceso de ajuste

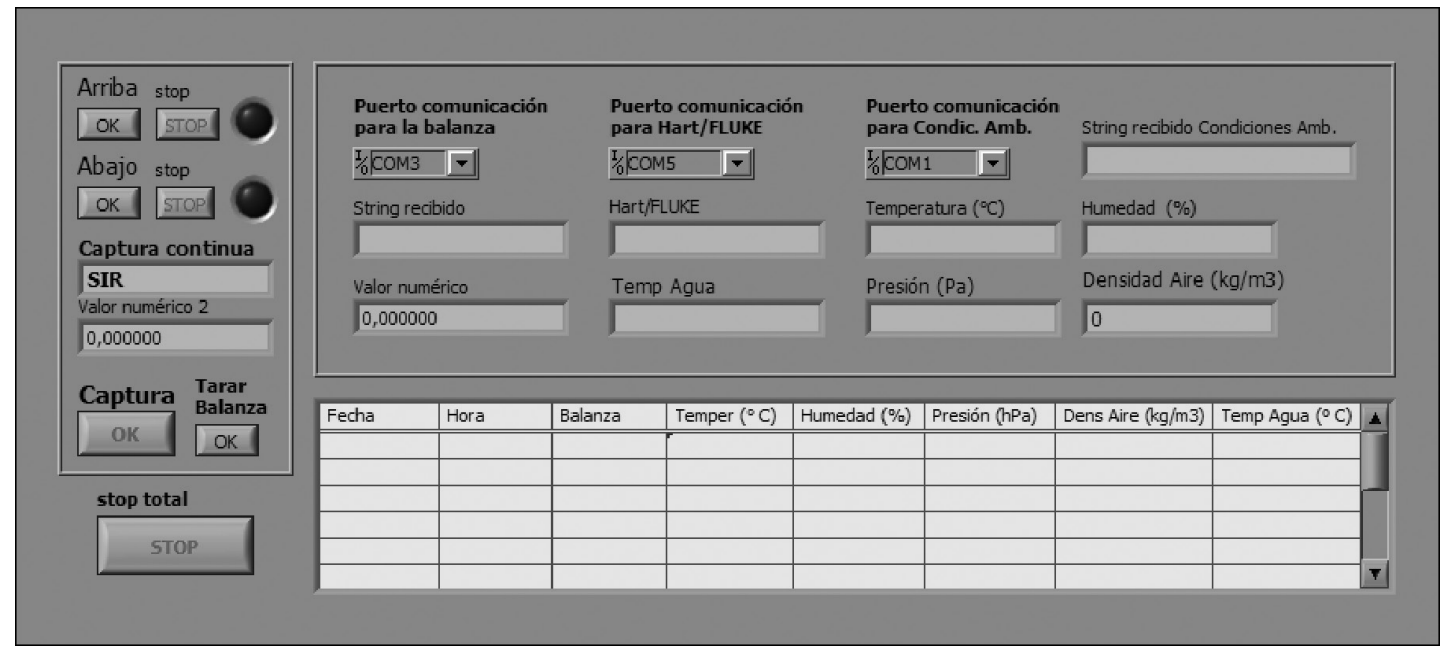

Figura13. Panel frontal para la calibración de un hidrómetro.

Fuente: (El autor). 


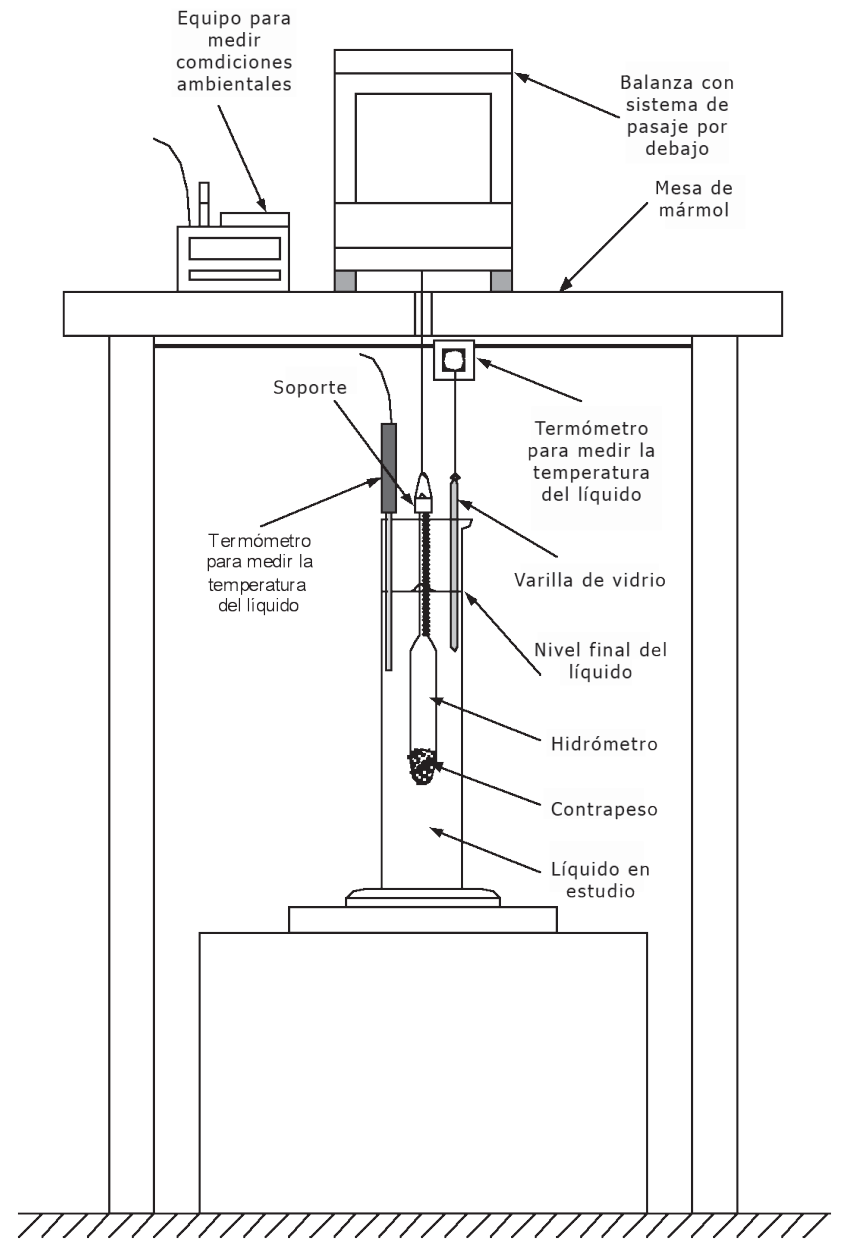

Figura 14. Montaje de equipos para la aplicación del Método de Cuckow para la calibración de hidrómetros con control semi- automatizado.

Fuente: (El autor).

del menisco es uno de los objetivos buscados e indicar la correcta frecuencia permite determinar la velocidad adecuada, esta velocidad debe ser lo suficientemente baja para que las vibraciones transmitidas a través del líquido de calibración sean apenas perceptibles por la balanza; si por otro lado se toma en cuenta que las distancias entre divisiones de escala en los hidrómetros son muy pequeñas, una velocidad suficientemente baja permitirá ajustar de forma más adecuada el nivel del líquido de calibración a la división de escala deseada.

Es importante tomar en cuenta que del mismo modo se puede producir un giro en el sentido inverso al modificarse el orden de energizado de los pines del puerto paralelo y por ende, de las bobinas del motor.

Una vez controlado el motor se pueden integrar más elementos al panel de control, esto significa que es probable recibir y colectar datos de otros equipos y controlar al mismo tiempo el motor, por ejemplo si se usa el puerto serial (RS232) de la PC es posible controlar la masa del hidrómetro al introducir o sacar la barra de vidrio del recipiente con una lectura directa de la balanza, además es factible obtener lecturas de condiciones ambientales en ese mismo instante.

Acceder al puerto serial es posible al usar las propiedades VISA de LabVIEW ${ }^{\circledR}$. 
Cada fabricante determina los parámetros particulares con los cuales sus equipos podrán transmitir datos con un protocolo RS232, por lo general estos parámetros pueden encontrarse en los manuales propios de cada equipo; habiendo determinado estos pueden crearse valores constantes como propiedades de la comunicación VISA, el puerto serial que será usado será determinado por la disponibilidad de puertos seriales de la PC.

La Figura 12 muestra la subrutina de comunicación para una balanza electrónica en donde cada parámetro VISA fue establecido por el fabricante, del mismo modo, cada parámetro de comunicación es propio del equipo. La idea es enviar una solicitud de información y como respuesta recibir un paquete de información, un string, que es manipulable e interpretable.

La posibilidad de colectar datos es bastante amplia, es incluso posible integrar y obtener datos de dos equipos al mismo tiempo, siempre y cuando se encuentren en puertos seriales diferentes.

Integrar todos los elementos necesarios para el proceso de calibración de hidrómetros es más sencillo entonces con el uso de LabVIEW $^{\circledR}$, el panel de control de la aplicación en estudio se verá de la siguiente forma (ver Figura 13).

El panel frontal de la aplicación para el proceso de calibración de hidrómetros con el uso del Método de Cuckow, tal y como se muestra en la Figura 13, integra cada elemento que puede afectar el proceso de calibración y que además pueda ser controlado con una PC; al lado izquierdo se ubica el control del motor junto con la lectura continua de la balanza y un botón de seguridad, del lado derecho se encuentran otros elementos como la balanza y equipos para la lectura y el registro de condiciones ambientales, cada dato es tabulado en una tabla en el momento en el que el usuario lo requiera, al final de todo el proceso los datos de esta tabla generan un informe en formato Excel ${ }^{\circledR}$ que puede ser usado como reporte de datos.

Todos estos elementos integrados en una sola aplicación agilizan el proceso de calibración de hidrómetros, sin embargo, las mejoras al proceso de calibración no solo dependen de la agilidad en la captura de datos, también depende de la forma en la cual se dispongan los equipos.

La Figura 14 presenta la correcta colocación del hidrómetro y de la barra de vidrio, en este caso el recipiente contenedor es una probeta la cual se coloca dentro de un baño termo estático que permita mantener la temperatura estable y controlada dentro de la probeta.

\section{RESULTADOS}

El proceso de calibración de hidrómetros presenta algunos requisitos y condiciones mínimas que deben ser considerados al ser desarrollado el método; estos requisitos, si son controlados de forma adecuada, permiten obtener resultados de calibración satisfactorios. Uno de los puntos más críticos por considerar en el proceso de calibración de hidrómetros es mantener estable durante el desarrollo de la prueba la temperatura del líquido de referencia en el que se calibra el instrumento. La forma en la que se realizaba el ajuste del menisco formado entre el líquido de referencia y el hidrómetro durante este proceso, es una de las limitantes que favorece los cambios en la temperatura en el líquido de referencia.

Antes de la introducción de mejoras en el proceso de calibración de hidrómetros realizado en el Laboratorio de Densidad del Laboratorio Costarricense de Metrología (LACOMET), el operario se mantenía cerca del recipiente que contenía el hidrómetro con el fin de ajustar el valor de escala agregando o quitando agua con un gotero o usando una gata de tijera, tal y como lo muestran las Figuras 2 y 3 , ambos métodos introducían serias perturbaciones al sistema y aumentaban los tiempos de calibración, lo que producía un aumento gradual en la temperatura en el líquido de referencia y un aumento de la inestabilidad de la balanza.

El aumento progresivo en la temperatura del líquido de referencia tiene dos efectos importantes sobre las mediciones y los resultados finales, primero un cambio en la tensión superficial del líquido de medición y 
Cuadro 2. Variación de la tensión superficial del agua debida a una variación en la temperatura.

Temperatura

$\left({ }^{\circ} \mathrm{C}\right)$

17

18

19

20

21

22
Tensión superficial del agua

$(\mathrm{N} / \mathrm{m})$

0,07319

0,07304

0,07289

0,07274

0,07258

0,07243
Incertidumbre
(N/m)
0,0037
0,0037
0,0037
0,0036
0,0036
0,0036

Fuente: (Inational Association for the Properties of Water and Steam (IAPWS) (1994), Release on Surface Tension of Ordinary Water Substance, Estados Unidos: IAPWS).

segundo, un cambio en la densidad del líquido de medición, lo que afecta el cálculo de la resolución del equipo.

\subsection{Cambios en la tensión superficial del líquido de referencia}

De acuerdo con Toledo (2001), Díaz y Becerra (2008), la tensión superficial es un fenómeno producido en el área en donde se encuentran un líquido y un gas que no son miscibles uno con el otro (la superficie del líquido) y que están en reposo, producido principalmente por las fuerzas de cohesión de las moléculas que forman los líquidos.

Internamente un líquido esta formado por moléculas sometidas a fuerzas de atracción y repulsión; en un nivel no superficial, el equilibrio de estas fuerzas es completo por lo que la interacción molecular es mínima y no existe inestabilidad, hay tal grado de equilibrio que el líquido tomará la forma del recipiente que lo contiene y mantendrá su forma siempre y cuando no haya cambios volumétricos importantes. A nivel superficial las moléculas del líquido se comportan diferente, las fuerzas de interacción entre estas moléculas están más equilibradas a nivel horizontal, ya que en este punto hay mayor interacción; arriba de la superficie del líquido no existe interacción con las moléculas de gas por lo que no hay equilibrio vertical, esta fuerza horizontal aumenta la resistencia a la rotura de la superficie y produce el efecto conocido como tensión superficial. La inestabilidad vertical produce que la tensión superficial sea una fuerza dirigida hacia el interior del recipiente y normal a la superficie del líquido, esto genera una curvatura hacia adentro del líquido en la superficie, la cual será el menisco.

Cuando ocurren cambios importantes en el comportamiento del líquido de referencia ocurrirán cambios en la tensión superficial y en la forma del menisco; al aumentar la temperatura las fuerzas de cohesión entre las moléculas que forman el líquido de referencia disminuyen, ya que aumenta el volumen del líquido, este cambio en el volumen y en las fuerzas de cohesión cambian la tensión superficial del líquido, este cambio en la tensión superficial cambia la forma del menisco, el resultado es un cambio en la forma en la que se observa el menisco y por ende, en la forma como se ajusta el punto de lectura.

El modelo matemático para la calibración de hidrómetros, tal y como lo muestra la ecuación (3), presenta dos valores de tensión superficial, la tensión superficial del líquido en el que se va a utilizar el hidrómetro $\left(\gamma_{x}\right)$ y la tensión superficial del líquido de medición $\left(\gamma_{L}\right)$, de acuerdo con Díaz y Becerra (2008) y de acuerdo con la ecuación (3), un cambio en la tensión superficial de $0,01 \mathrm{~N} / \mathrm{m}$ producirá un cambio en el valor de la densidad leída de $0,1 \mathrm{~kg} / \mathrm{m}^{3}$ lo que para densímetros con resolución de $0,01 \mathrm{~kg} / \mathrm{m}^{3}$ es un cambio significativo que debe ser tomado en cuenta. 
Cuadro 3. Variación de la densidad del agua debida a una variación en la temperatura.

\section{Temperatura}

$\left({ }^{\circ} \mathrm{C}\right)$

17

18

19

20

21

22
Densidad del agua
$\left(\mathrm{g} / \mathrm{cm}^{3}\right)$
0,99877
0,99860
0,99840
0,99820
0,99799
0,99777

Fuente: (Becerra y Sandoval, 2002; "Determinación de la densidad en líquidos y sólidos", CENAM, México, CNM-MMM-PT-002, pp. 63-64).

Cuadro 4. Comparación de datos de temperatura durante el proceso de calibración de un hidrómetro.

\begin{tabular}{|c|c|c|c|c|}
\hline & \multicolumn{4}{|c|}{ Comparación de datos de temperatura durante la calibración } \\
\hline & \multicolumn{2}{|c|}{2006} & \multicolumn{2}{|c|}{2009} \\
\hline & $\begin{array}{c}\text { Temperatura del } \\
\text { agua }\end{array}$ & Densidad del agua & $\begin{array}{c}\text { Temperatura del } \\
\text { agua }\end{array}$ & Densidad del agua \\
\hline & $\left({ }^{\circ} \mathrm{C}\right)$ & $\left(\mathrm{g} / \mathrm{cm}^{3}\right)$ & $\left({ }^{\circ} \mathrm{C}\right)$ & $\left(\mathrm{g} / \mathrm{cm}^{3}\right)$ \\
\hline & 19,502 & 0,998305 & 20,168 & 0,998161 \\
\hline & 19,532 & 0,998299 & 20,193 & 0,998155 \\
\hline & 19,647 & 0,998276 & 20,214 & 0,998151 \\
\hline & 19,746 & 0,998255 & 20,222 & 0,998149 \\
\hline & 19,893 & 0,998225 & 20,273 & 0,998139 \\
\hline Promedio: & 19,664 & 0,998272 & 20,214 & 0,998151 \\
\hline ón estándar: & 0,161 & 0,000033 & 0,039 & 0,000008 \\
\hline
\end{tabular}

Fuente: (Rodríguez, 2009; Laboratorio de Densidad, LACOMET).

El Cuadro 2 muestra los valores en los que varía la tensión superficial con respecto a la temperatura de uno de los líquidos de referencia más comúnmente usados para la calibración de hidrómetros, el agua.

Los datos del Cuadro 2 están basados en la revisión de los resultados de la medición de la tensión superficial realizados por el Grupo de Trabajo III de la IAPWS de los datos y los resultados de 1975 para la tensión superficial del agua, los cuales estaban basados en la ITPS-68, la revisión de 1994 actualizó estos resultados a la ITS-90 con base en la siguiente ecuación.

$$
\gamma=B \tau^{\mu}(1+b \tau)
$$

En donde:

$\begin{array}{ll}\gamma & \text { tensión superficial } \\ \tau & \left(1-\mathrm{T} / \mathrm{T}_{\mathrm{c}}\right) \\ \mathrm{T} & \text { temperatura }(\mathrm{K}) \\ \mathrm{T}_{\mathrm{c}} & 647,096 \mathrm{~K} \\ \mathrm{~B} & 235,8 \mathrm{mN} / \mathrm{m} \\ \mathrm{b} & -0,625 \\ \mu & 1,256\end{array}$


in

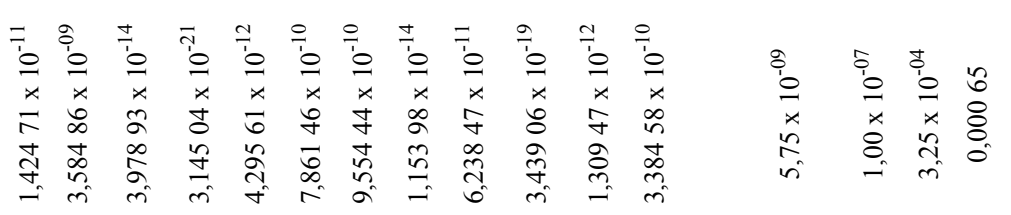

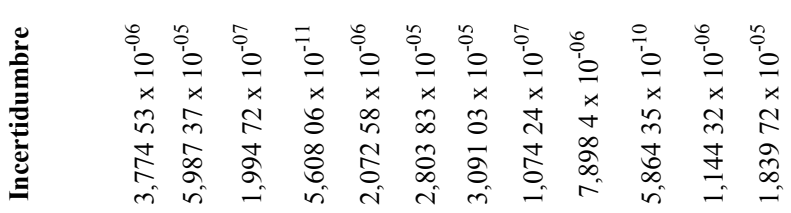

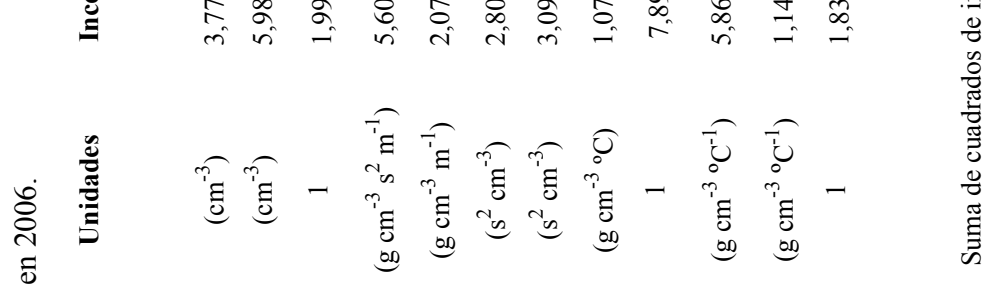

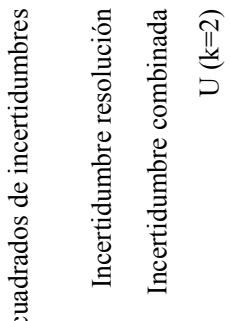

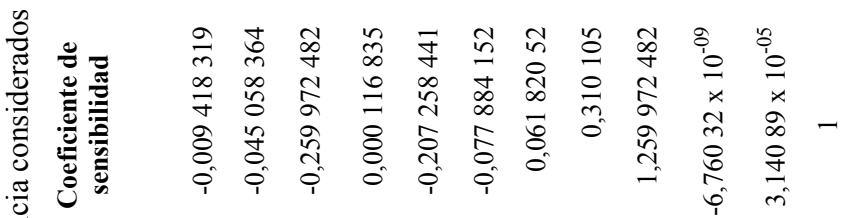

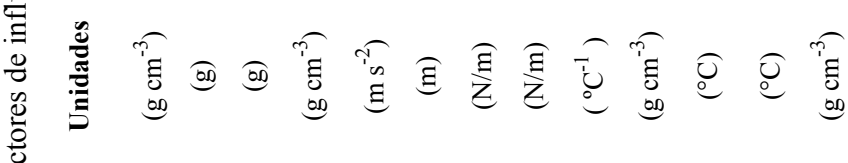

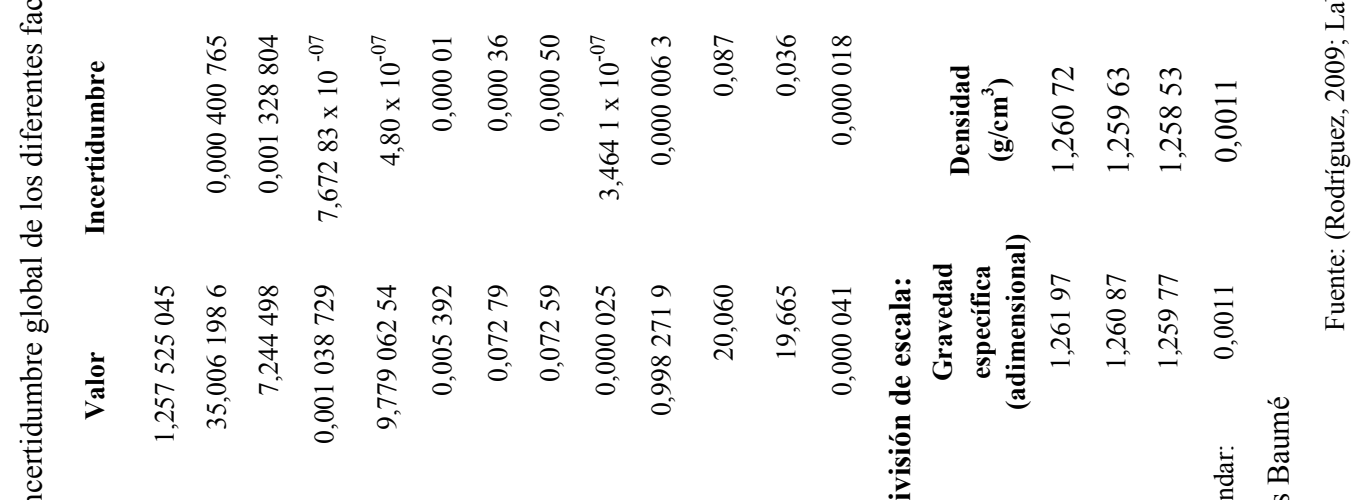

.

के

$\frac{\pi}{8}$

离

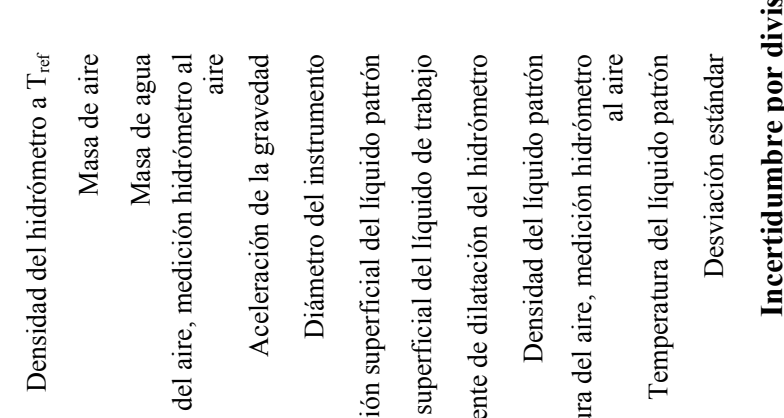

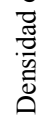

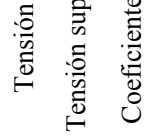




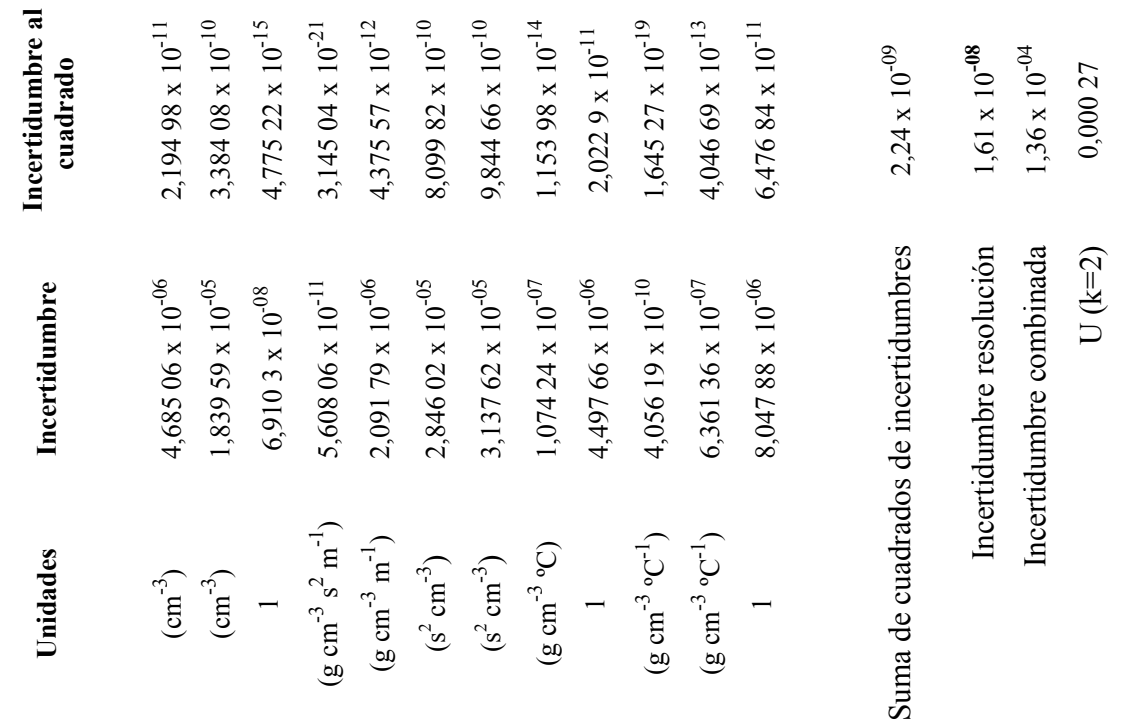

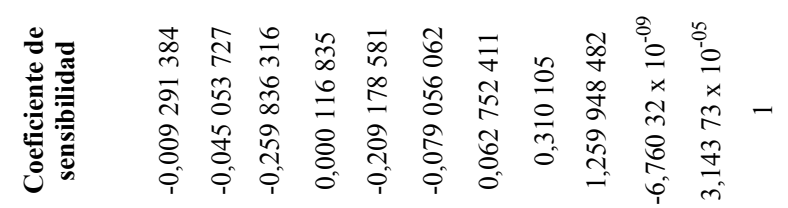

竞

荧

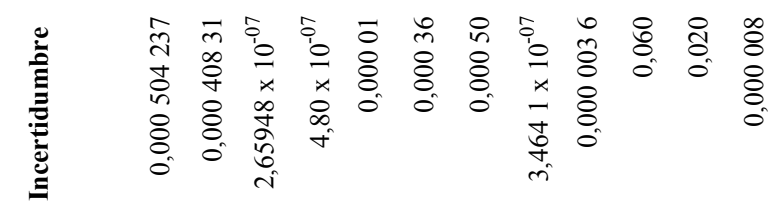

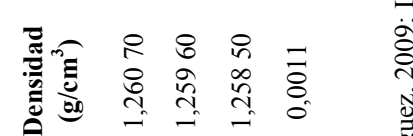

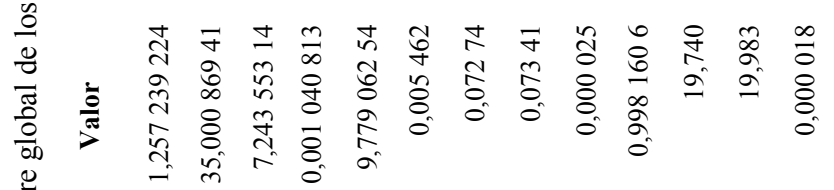

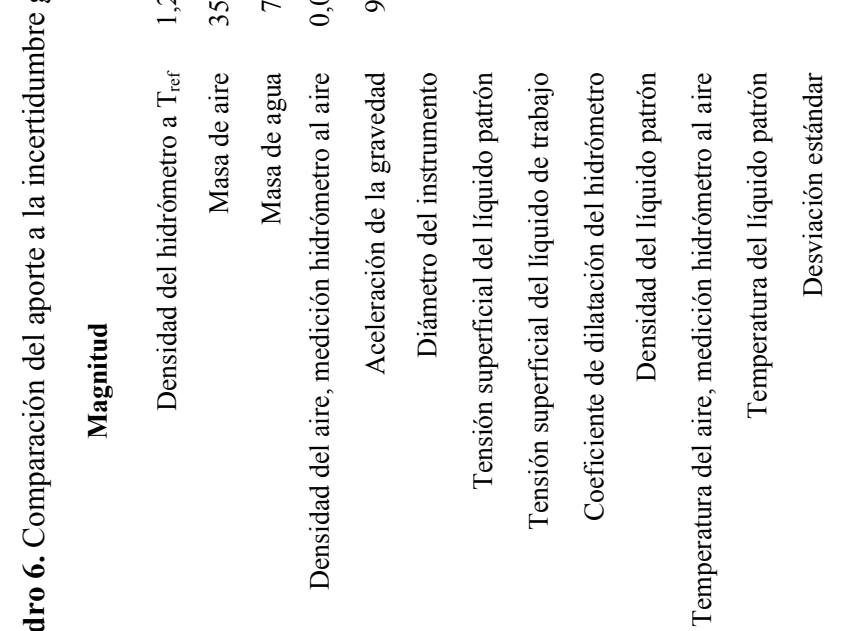

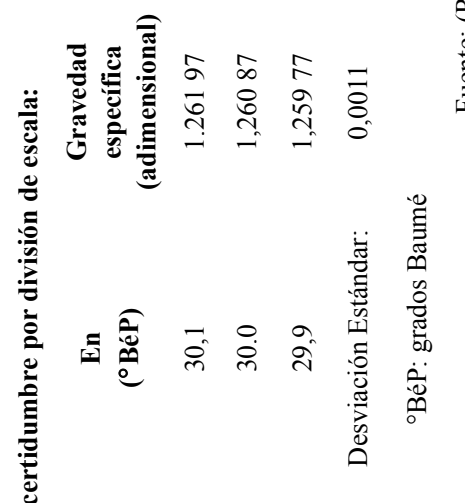

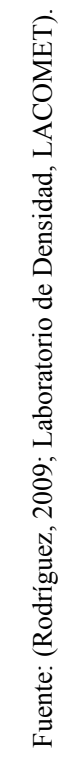




\subsection{Cambios en la densidad del líquido de referencia}

Tal y como se discutió anteriormente, internamente un líquido está formado por moléculas sometidas a fuerzas de atracción y repulsión, a un nivel no superficial; el equilibrio de estas fuerzas es completo por lo que la interacción molecular es mínima y no existe inestabilidad, hay tal grado de equilibrio que el líquido tomará la forma del recipiente que lo contiene y mantendrá su forma, esta característica determinará el volumen del líquido de referencia.

Cuando un hidrómetro es sumergido en el líquido de referencia hasta una marca de lectura determinada y ambos, líquido e instrumento, son llevados al equilibrio el peso será igual al empuje.

$m g=\rho_{L} V g$

En donde:

$\begin{array}{ll}m & \text { masa del hidrómetro } \\ g & \text { aceleración de la gravedad } \\ \rho_{L} & \text { densidad del líquido de referencia } \\ V & \text { volumen del líquido }\end{array}$

La forma en la que se determina la densidad del líquido de referencia generalmente usado para calibrar hidrómetros, agua, está determinada por la ecuación 9. Tomando en cuenta la ecuación 9 y 19 se determina que la densidad está afectada por los cambios en la temperatura de tal forma que un cambio en $0,1^{\circ} \mathrm{C}$ en la temperatura del agua producirá un cambio aproximado en la densidad del líquido de referencia de entre 0,01 $\mathrm{kg} / \mathrm{m}^{3}$ a $0,02 \mathrm{~kg} / \mathrm{m}^{3}$, este cambio en la densidad afectará de forma sensible los valores de lectura de hidrómetros con resoluciones similares o menores a $0,01 \mathrm{~kg} / \mathrm{m}^{3}$, en especial en lugares en donde la temperatura del líquido referencia no es estable.

El Cuadro 3 muestra los valores en los que varía la densidad del líquido con respecto a la temperatura, de uno de los líquidos de referencia más comúnmente usados para la calibración de hidrómetros, el agua.

Los Cuadros 2 y 3 muestran las variaciones producidas en la tensión superficial y en la densidad del agua, fluido comúnmente usado para la calibración de hidrómetros, por cada grado Celsius; con esta información es posible percibir efectos importantes en los resultados de medición de la densidad del líquido con el uso de un hidrómetro en particular debidos a cambios significativos en la temperatura del líquido de referencia utilizado.

\subsection{Resultados comparativos de la calibración de un hidrómetro antes y después de implementado el sistema semi- automatizado de ajuste y de adquisición de datos en el LACOMET}

Una comparación de los datos generados en la calibración de un mismo hidrómetro en el 2006, en donde el ajuste del menisco se hizo por métodos tradicionales, contra resultados de calibración de 2009, en donde el ajuste del menisco se hizo con la ayuda de una barra de vidrio con ascenso y descenso controlado, permite observar los cambios significativos en la estabilidad del medio de calibración.

El Cuadro 4 presenta los datos obtenidos en la calibración de un hidrómetro, en un mismo punto de calibración, en donde es posible observar las variaciones significativas en el comportamiento de la temperatura durante la calibración de 2006, lo cual se comprueba con la desviación estándar de estos datos, por otro lado, se puede observar la variación en el comportamiento de la densidad del agua al observar el cambio en la desviación estándar durante el proceso. Por el contrario, los datos del 2009, y gracias a la estabilidad que existió entre el medio de calibración y el medio de ajuste, y a la reducción en el tiempo de ajuste ofrecida por este sistema más controlado, la temperatura mostró una menor variación; las desviaciones estándar muestran que la tendencia hacia un sistema más estable contribuyó a una determinación más óptima de la densidad del medio de calibración, esto aportó un menor valor a la incertidumbre por la temperatura y por la densidad del líquido de referencia en los resultados obtenidos en el 2009. 
Una determinación más precisa de la densidad del líquido permite una mejor determinación de la resolución del instrumento calibrado, ya que es necesario este valor de densidad para realizar este cálculo a instrumentos con escala en unidades diferentes a las del SI. El hidrómetro calibrado en el 2006 y en el 2009 presenta una escala de medición en unidades diferentes a las del SI.

$$
\rho_{x}=\left(\frac{145}{145-\left({ }^{\circ} B \dot{P} P\right)}\right) \rho_{L}
$$

En donde:

$\rho_{x} \quad$ densidad del hidrómetro en unidades del $\mathrm{SI}\left(\mathrm{kg} / \mathrm{m}^{3}\right)$

$\rho_{L} \quad$ densidad del líquido de referencia en unidades del SI $\left(\mathrm{kg} / \mathrm{m}^{3}\right)$

${ }^{\circ}$ BéP valor de la escala del hidrómetro en grados Baumé Pesados

La ecuación (20) muestra la forma en la que se realiza la conversión para hidrómetros en grados Baumé Pesados, que es el caso en estudio, el valor equivalente a la densidad en unidades del SI dependerá del valor de la densidad del líquido de referencia. Un análisis de la incertidumbre aportada por el valor calculado de la resolución del instrumento permite establecer el aporte a la incertidumbre global en el 2006 y en el 2009.

En los Cuadros 5 y 6 se muestran los aportes a la incertidumbre global de los diferentes factores de influencia en las calibraciones realizadas en el 2006 y en el 2009 para el mismo hidrómetro en el mismo punto de medición; es evidente que la resolución aporta la mayor parte de la incertidumbre y que la forma en la que la resolución sea determinada con base en la densidad del líquido de referencia y la forma en la que la que la densidad sea determinada por las lecturas realizadas, determinan los valores finales de incertidumbre.

\section{CONCLUSIONES}

1. La introducción de un método semiautomatizado de ajuste del menisco redujo el contacto entre el medio de calibración y el operario y los tiempos en el proceso de ajuste del menisco, lo cual mejora la estabilidad del medio por la disminución en las perturbaciones y en el contenido de burbujas del sistema, además se favoreció la reducción de los gradientes de temperatura durante el proceso; del mismo modo, la reducción en los tiempos de ajuste favoreció la disminución en las variaciones en la densidad del líquido de calibración.

2. La implementación de este sistema semiautomatizado de ajuste del menisco ha demostradoserunaherramientabeneficiosa en el desarrollo de las calibraciones de hidrómetros al favorecer una reducción en la incertidumbre por tensión superficial y por variaciones en la densidad del agua durante el proceso.

3. La implementación de este sistema semiautomatizado de ajuste del menisco y a su vez, la integración y el control de los equipos involucrados en una sola aplicación desarrollada con el software LabVIEW $^{\circledR}$ ha demostrado ser una herramienta de fácil uso que no solo mejora los tiempos de calibración, sino que también ha permitido acceder a la información de forma directa con una captura confiable de datos y la generación de un reporte de calibración confiable y ágil, por completo utilizable como documento de referencia, esto también favorece los tiempos finales de respuesta hacia los clientes con un análisis de datos y una emisión de resultados más rápida.

4. Una de las ventajas de integrar diferentes equipos y de realizar la adquisición de datos de forma semi-automatizada es la rapidez del proceso y la confiabilidad de los resultados obtenidos y almacenados, con lo que se evita la toma de datos manual y la manipulación de los resultados. 


\section{AGRADECIMIENTOS}

Quiero agradecer a la Lic. Sandra Rodríguez Zúñiga y al B.F. Adrián Solano Mena, ambos miembros del personal técnico de LACOMET por su ayuda, colaboración, apoyo y sugerencias en el desarrollo de este artículo.

\section{REFERENCIAS BIBLIOGRÁFICAS}

Becerra, L. O. \& Sandoval, F. P. (2002). Determinación de la densidad en líquidos y sólidos, CNM-MMM-PT-002, México: CENAM.

Bitter, R.; Mohiunddin, T. \& Nawrocki, M. (2007). LabVIEW, Advanced Programming Techniques. Florida: Taylor \& Francis Group.

Díaz, J. C. \& Becerra, L. O. (2008). Tensión superficial. Importancia de las mediciones en la metrología de densidad con un valor de incertidumbre aceptable, Centro Nacional de metrología de México. En: Simposio de Metrología 2008, SM2008-M108-1050-1, CENAM.

García, F. (2006). Documento técnico: Manejo de hidrómetros, División de Metrología - CESMEC Ltda. Extraído el 12 de enero de 2009, de: http://www. metrologia.cl/medios/hidrometro.pdf

Gupta, S. V. (2002). Practical density measurement and hydrometry. London: Institute of Physics Publishing.

Harries, I. (2006). Stepper motor controller connection diagrams. Extraído el 23 de octubre de 2008, de: http://www. doc.ic.ac.uk/ ih/doc/stepper/control2/ connect.html

International Association for the Properties of Water and Steam (IAPWS). (1994). release on surface tension of ordinary water substance. Estados Unidos: IAPWS.
Leandro, G. (1998). Medición de la gravedad observada en ONNUM y Escuela de Física / UCR, Reporte Técnico de la Oficina de Geofísica Aplicada del ICE, Costa Rica: ICE-ONNUM-UCR, p. 14.

Peña, L. M.; Pedraza, J. C. \& Becerra, L. O. (2007). A new image system for hydrometer calibration developed at CENAM, Centro Nacional de Metrología de México. En: IMEKO $20^{\text {th }}$ TC3, $3^{\text {rd }}$ TC16 and $1^{\text {st }}$ TC22, International Conference.

Santo, C. \& Cáceres, J. (2006). Método de Cuckow para la calibración de aerómetros utilizando agua destilada adicionada con un tensoactivo como fluido patrón, Laboratorio Tecnológico del Uruguay - LATU. En: Simposio de Metrología 2006, CENAM.

Sinclair, I. \& Duton, J. (2007). Practical electronics handbook. England: NewnwsElsevier, p. 139, 191-193, 198, 233, 379.

Sumathi, S. \& Surekha, P. (2007). LabVIEW, Based advanced instrumentation systems. Berlin: Springer-Verlag.

Toledo,C. (2001).Conceptos básicos de mecánica de fluidos, programa de perfeccionamiento fundamental. Universidad de Santiago de Chile. Extraído el 12 de enero de 2009, de: http://fisica.usach.cl/ didactic/fluidos_uno_ cecilia.pdf

\section{SOBRE EL AUTOR}

\section{Olman Fernando Ramos Alfaro}

Jefe del Laboratorio de Masas del Laboratorio Costarricense de Metrología (LACOMET)

Colaborador en los Laboratorios de Densidad, Volumen y Presión de LACOMET Teléfono: (506) 2283 6580/ (506) 22805387, extensiones: $111 / 112$

Facsímil: (506) 22835133

Correo electrónico: olmanramos@gmail.com / oramos@lacomet.go.cr 

Notas técnicas 
